\title{
Morse Index and Stability of the Planar N-vortex Problem
}

\author{
Xijun $\mathrm{Hu}^{1} \cdot$ Alessandro Portaluri $^{2}$ (D) Q Qin Xing ${ }^{1}$ \\ Received: 6 June 2019 / Accepted: 12 July 2020 / Published online: 3 August 2020 \\ (c) The Author(s) 2020
}

\begin{abstract}
This paper concerns the investigation of the stability properties of relative equilibria which are rigidly rotating vortex configurations sometimes called vortex crystals, in the $\mathrm{N}$-vortex problem. Such a configurations can be characterized as critical point of the Hamiltonian function restricted on the constant angular impulse hyper-surface in the phase space (topologically a pseudo-sphere whose coefficients are the circulation strengths of the vortices). Relative equilibria are generated by the circle action on the so-called shape pseudo-sphere (which generalize the standard shape sphere appearing in the study of the $\mathrm{N}$-body problem). Inspired by the planar $\mathrm{N}$-body problem, and after a geometrical and dynamical discussion of the problem, we investigate the relation intertwining the stability of relative equilibria and the inertia indices of the central configurations generating such equilibria. In the last section we applied our main results to some symmetric three and four vortices relative equilibria.
\end{abstract}

Keywords Relative equilibria $\cdot \mathrm{N}$-vortex problem $\cdot$ Spectral stability $\cdot$ Morse index

Mathematics Subject Classification 70H14 · 70F15 - 37J25 · 34D20 · 37N10 · 76B47

X. Hu: Partially supported by NSFC (Nos. 11790271, 11425105). A. Portaluri: Partially supported by Prin 2015: Variational methods, with applications to problems in mathematical physics and geometry.

$凶 \quad$ Alessandro Portaluri

alessandro.portaluri@unito.it

Xijun $\mathrm{Hu}$

xjhu@sdu.edu.cn

Qin Xing

qinxingly@gmail.com

1 School of Mathematics, Shandong University, Jinan 250100, Shandong, People's Republic of China

2 DISAFA, Università degli Studi di Torino, Largo Paolo Braccini 2, 10095 Grugliasco, Turin, Italy 


\section{Introduction and Description of the Problem}

The study of vortex dynamics can be traced back to Helmholtz's work on hydrodynamics in 1858 [1] and it plays an important role in the study of superfluids, superconductivity, and stellar systems. Its Hamiltonian formulation could be dated back to Kirchhoff in the plane, and later on generalized by Routh [2] and then Lim [3] to general domains in the plane. In this paper, we are interested to the problem in the first order Hamiltonian system of the form

$$
\Gamma_{i} \dot{z}_{i}(t)=J \nabla_{z_{i}} H(z(t)) \quad i \in 1, \ldots, N
$$

Here $J:=\left[\begin{array}{ll}0 & 1 \\ -1 & 0\end{array}\right]$ is the standard symplectic matrix in the Euclidean plane. The Hamiltonian function $H$ is

$$
H(z)=-\sum_{\substack{i, j=1 \\ i<j}}^{N} \Gamma_{i} \Gamma_{j} \log \left|z_{i}-z_{j}\right|
$$

Here $\Gamma_{1}, \ldots \Gamma_{N} \in \mathbb{R} \backslash\{0\}$ are the vorticities or vortex strengths. The Hamiltonian it is defined on the configuration space

$$
\mathbb{F}_{N}\left(\mathbb{R}^{2}\right):=\left\{z \in \mathbb{R}^{2 N} \mid z_{i} \neq z_{j} \text { for } i \neq j\right\}
$$

of the $N$ (coloured) points in the plane. It is clear by the definition that $H\left(z_{1}, \ldots, z_{N}\right)$ becomes singular if $\left|z_{i}-z_{j}\right| \rightarrow 0$ for some $i \neq j$. Setting $G\left(\boldsymbol{w}_{1}, \boldsymbol{w}_{2}\right)=$ $-\log \left|\boldsymbol{w}_{1}-\boldsymbol{w}_{2}\right|$ then the Hamiltonian can also be written as $H(\boldsymbol{z})=\sum_{i<j} \Gamma_{i} \Gamma_{j} G$ $\left(\boldsymbol{z}_{i}, \boldsymbol{z}_{j}\right)$ and it is usually called hydrodynamic Green's function. As already observed, the Hamiltonian system in Eq. (1.1) appear as singular limit equations in problems from physics. More precisely in fluid dynamics is derived from the Euler equation and for instance in superconductivity $H$ appears as renormalized energy for Ginzburg-Landau vortices. Concerning the existence and stability properties of periodic solutions of the $\mathrm{N}$-vortex problem given in Eq. (1.1), the literature is quite broad and we refer the interested reader to [4] and references therein. Among the simplest periodic orbits of the planar $N$-vortex problem are the relative equilibria. These configurations of vortices rotates rigidly about their center of vorticity and sometimes are referred to as vortex crystals and are frequently observed in natural phenomena (e.g. the hurricanes).

Relative equilibria are crucial in deeply understanding the intricate dynamics of this singular Hamiltonian problem and as the name suggested are rest-points in a suitable rotating coordinate system. As we'll discuss in Sect. 2, relative equilibria can be characterized as critical points (or more precisely critical orbits) of the restriction of the Hamiltonian to the angular impulse unitary (pseudo-)sphere of the phase space. Otherwise stated such a rigid configurations are generated through a rotation with angular velocity $\omega$ of a special critical configuration of the system usually called central configuration. (Cfr. Sect. 2 for further details). 
A natural and classical problem is to understand how the spectral properties of these central configurations or more precisely the inertia indices of the Hessian of $H$ at these configurations reflect on the dynamical properties of the generated vortex crystal (through rotation) like, for instance, spectral or linear stability properties etc. This problem is very classical in the gravitational $N$-body problem in which central configurations are characterized as critical points of the self-interacting potential on the shape sphere (which is the base space of the circle bundle) whose total space is the inertia ellipsoid. There is a long standing conjecture due to Moeckel stating that a linearly stable relative equilibrium must be a nondegenerate minimum of the Newtonian potential restricted to the shape sphere. The other direction is false even for other class of weakly attracting singular potentials. (Cfr., for instance, [5,6]).

The investigation of the relation between the stability properties of a relative equilibrium and the spectral properties of the central configuration generating such an equilibrium in the N-vortex problem is pretty different. Despite of the fact that the circulation strengths could have any sign (in the classical gravitational $N$-body problem they correspond to the masses which are all positive), the Hessian of the Hamiltonian computed at a central configuration has some commutativity properties with respect to the Poisson matrix $K$ induced by $J$ that greatly simplify the problem. (Cfr. Lemma 2.6, for further details). Such a property was observed by Roberts in its interesting paper of Roberts [7,8]. In the aforementioned papers, in fact, the author was able among others to prove in the case of positive circulation strengths, the relation between the Morse index of a relative equilibrium $z$ and the spectrum of the so-called stability matrix, i.e. the matrix which determines the linear stability of $z$. (Cfr. [8, Theorem 3.5] and references therein). As direct consequence he proved (always under the assumption of all positive circulations) that a relative equilibrium $z$ of the $N$-vortex problem is linearly stable if and only if it is generated by a nondegenerate minimum of the Hamiltonian $H$ restricted to the level surface of the angular impulse.

This result was the starting point of our analysis and the main motivation to investigate about the effect of mixed sign circulation strengths. Last but not least comment before describing our main results is to observe that this indefinite case, the stability analysis is much more delicate. This situation, as we'll try to clarify, reflects somehow the difficulties and it is the paradigm of the difference between the Riemannian and the Lorentzian world.

\subsection{Main Results}

Our first result, provides a characterization of the spectral stability of a relative equilibrium $z$ in terms of a spectral condition on the central configuration $\xi$, no matter how the signs of the circulations are. Before stating and describing our first result, we pause by recalling what stability notion we are talking about. Being the Hamiltonian $H$ invariant under translations and rotations this implies, among others, that 0 (having algebraic multiplicity 2) as well as $\pm \omega i$ are Floquet characteristic multipliers, arising precisely from these symmetries.

What is natural to do is to define the linear stability properties of a relative equilibrium by ruling out the eigenvalues coming from these conservation laws. We set 
$M_{\Gamma}:=\Gamma_{i} I_{2} \delta_{i j}$ (where $I_{2}$ denotes the $2 \times 2$ identity matrix and $\delta_{i j}$ is the Kronecker delta) and we denote by $B$ the so-called stability matrix, namely the matrix which is responsible of the stability of the relative equilibrium which is defined by $B=K\left[M_{\Gamma}^{-1} D^{2} H(\xi)+\omega I_{2 N}\right]$ where $K$ is the Poisson matrix, i.e. a $2 \times 2$ block diagonal matrix in which each non-vanishing block is given by $J$.

Definition 1.1 A relative equilibrium $z$ will be termed non-degenerate provided the remaining $2 n-4$ eigenvalues of the matrix $B$ are not vanishing. A non-degenerate relative equilibrium is

- Spectrally stable if the nontrivial eigenvalues are purely imaginary

- Linearly stable if, in addition, the restriction of the stability matrix $B$ to $W^{\perp}$ has a block-diagonal Jordan form with blocks $\left[\begin{array}{cc}0 & \beta_{i} \\ -\beta_{i} & 0\end{array}\right]$.

Remark 1.2 Otherwise stated a relative equilibrium $z$ is spectrally stable if all Floquet multipliers (the eigenvalues of the monodromy matrix) belongs to the unit circle (centered at the origin) $\mathbb{U}$ in the complex plane. Furthermore if the monodromy matrix is also diagonalizable, then $z$ is linearly stable. In this last case, in fact, the monodromy matrix can be factorized as direct symplectic sum of rotations or which is the same, it belongs to the maximal compact Lie subgroup of $\operatorname{Sp}(2 N-4)$.

Theorem 1 A non-degenerate relative equilibrium $z$ (with angular velocity $\omega$ ) generated by the central configuration $\xi$ is spectrally stable if and only iffor every eigenvalue $\mu$ of $M_{\Gamma}^{-1} D^{2} H(\xi)$ one of the following alternative holds

- $\mu \in i \mathbb{R}$

- $\mu \in \mathbb{R}$ and $\mu \in[-|\omega|,|\omega|]$.

In particular it is non-degenerate if and only if $\mu \neq \pm \omega$.

The idea for proving this result is essentially based on the relation between the matrix $M_{\Gamma}^{-1} D^{2} H(\xi)$ and $B$. It is worth to observe that, no matter how the signs of the circulations are, the matrix appearing in Theorem 1 i.e. $M_{\Gamma}^{-1} D^{2} H(\xi)$ is $M_{\Gamma}$-symmetric namely is symmetric with respect to the scalar product induced by $M_{\Gamma}$ (cfr. Definition 2.12 for further details). However, if the circulations are all positive such a scalar product is positive definite and this implies that the spectrum of $M_{\Gamma}^{-1} D^{2} H(\xi)$ is diagonalizable in the orthogonal group and its spectrum is real. For mixed signs circulations, this is not true anymore, and in fact the spectrum of $M_{\Gamma}^{-1} D^{2} H(\xi)$ will be, in general, not real anymore. This reflects the indefinite Krein structure behind and among others responsible of the presence of Jordan blocks that are intimately related to the spectral stability properties of the relative equilibrium. In conclusion Theorem 1 represents the generalization of [7, Theorem 3.1] in the case of mixed signs circulations. The matrix $B$ is $M_{\Gamma^{-}}$-Hamiltonian, namely is Hamiltonian with respect to the symplectic form $\omega_{\Gamma}$ which is represented by $K$ with respect to the $M_{\Gamma}$-scalar product.

Our second main result relates the spectrally stability properties of a relative equilibrium and the inertia indices of the central configuration generating it.

Theorem 2 Let $z$ be non-degenerate relative equilibrium generated by the central configuration $\xi$ and let $\widehat{A}(\xi):=D^{2} H(\xi)+\omega M_{\Gamma}$. We assume that $z$ is spectrally stable. Then the following result holds. 


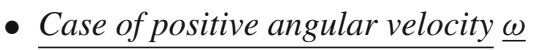

$$
\begin{cases}\mathrm{n}_{-}(\widehat{A}(\xi))=\mathrm{n}_{-}\left(M_{\Gamma}\right), & \text { if }\left\langle M_{\Gamma} \xi, \xi\right\rangle \text { is positive definite } \\ \mathrm{n}_{-}(\widehat{A}(\xi))=\mathrm{n}_{-}\left(M_{\Gamma}\right)-1, & \text { if }\left\langle M_{\Gamma} \xi, \xi\right\rangle \text { is negative definite. }\end{cases}
$$

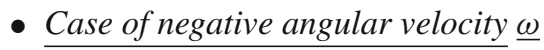

$$
\begin{cases}\mathrm{n}_{-}(\widehat{A}(\xi))=\mathrm{n}_{+}\left(M_{\Gamma}\right)-1, & \text { if }\left\langle M_{\Gamma} \xi, \xi\right\rangle \text { is positive definite } \\ \mathrm{n}_{-}(\widehat{A}(\xi))=\mathrm{n}_{+}\left(M_{\Gamma}\right), & \text { if }\left\langle M_{\Gamma} \xi, \xi\right\rangle \text { is negative definite }\end{cases}
$$

Furthermore, we have

$$
\begin{cases}\mathrm{n}_{-}\left(\left.\widehat{A}\right|_{W^{\perp}}(\xi)\right)=\mathrm{n}_{-}\left(\left.M_{\Gamma}\right|_{W^{\perp}}\right), & \text { if } \omega>0 \\ \mathrm{n}_{-}\left(\left.\widehat{A}\right|_{W^{\perp}}(\xi)\right)=\mathrm{n}_{+}\left(\left.M_{\Gamma}\right|_{W^{\perp}}\right), & \text { if } \omega<0\end{cases}
$$

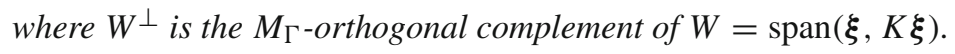

Remark 1.3 Theorem 2, in the case of mixed-sign circulations, was quite unexpected. This fact, as already confirmed by [8, Remark 4.10] in which author guess is that no connections seems existing between the Morse index of a central configuration and the stability of the arising relative equilibrium.

However, in Theorem 2, irrespective on what the signs of circulations are and by using a $M_{\Gamma}$-orthogonal decomposition, we were able to point out the deep relationship between the inertia indices of $M_{\Gamma}$ and the stability.

Given $N$-vortices in the plane, we define total vortex angular momentum $L$ as $L:=$ $\sum_{i<j} \Gamma_{i} \Gamma_{j}$. Thus, if the vortex strengths are all positive, then $L>0$. However, when vorticities are different in signs, then $L$ could be of any sign or even vanishes. Analogously to the moment of inertia in the $N$-body problem, it is possible to define the so-called angular-impulse of the $N$-vortex problem as follows

$$
I(z):=\frac{1}{2} \sum_{i=1}^{N} \Gamma_{i}\left\|z_{i}\right\|^{2}
$$

and, as we will see in the sequel, it will be crucial in order to give a variational interpretation to the central configurations, miming the analogous interpretation in the $N$-body problem. As already observed, for a relative equilibrium $z$ generated by $\xi$, the angular velocity is constant and it is given by

$$
L=\omega\langle\nabla I(\boldsymbol{\xi}), \boldsymbol{\xi}\rangle=2 \omega I(\boldsymbol{\xi}) \quad \Rightarrow \quad \omega=L /(2 I(\boldsymbol{\xi})) .
$$

Thus in the case of mixed signs circulations the angular velocity could have any sign what that cannot happen in the case of constant sign circulations.

The proof of Theorems 1 and 2 will be given in Sect. 4 . 
In the last section, we analyze some interesting symmetric central configuration; more precisely, the equilateral triangle and the rhombus (sometimes called kite) central configuration.

The equilateral triangle central configuration in the three-vortex problem is obtained by placing three vortices of any strength at the vertices of an equilateral triangle. Synge in his celebrated paper published in 1949 (cfr. [9]), proved that the corresponding relative equilibrium is linearly stable if and only if $L>0$.

Starting from this we get information on the a central configuration knowing the stability of the induced relative equilibrium. More precisely, let us given three circulations $\Gamma_{1}, \Gamma_{2}, \Gamma_{3}$ placed at the following points

$$
\widehat{\boldsymbol{\xi}}_{1}=(1,0), \widehat{\boldsymbol{\xi}}_{2}=\left(-\frac{1}{2}, \frac{\sqrt{3}}{2}\right), \widehat{\boldsymbol{\xi}}_{3}=\left(-\frac{1}{2},-\frac{\sqrt{3}}{2}\right),
$$

and we let $\widehat{\boldsymbol{c}}=\sum_{i=3}^{3} \Gamma_{i} \widehat{\boldsymbol{z}}_{i}$. Assuming that $L>0$ and setting $\boldsymbol{\xi}=\left(\boldsymbol{\xi}_{1}, \boldsymbol{\xi}_{2}, \boldsymbol{\xi}_{3}\right)$, for $\boldsymbol{\xi}_{i}=\widehat{\boldsymbol{\xi}}_{i}-\widehat{\boldsymbol{c}}$ then we conclude that

$$
\mathrm{n}_{-}\left(\widehat{A}_{\Gamma}(\xi)\right)=\left\{\begin{array}{ll}
0 & \text { if } \Gamma_{1}, \Gamma_{2}, \Gamma_{3} \text { have the same sign } \\
1 & \text { if there is only one } \Gamma_{i}<0 \\
2 & \text { otherwise }
\end{array} .\right.
$$

About the kite central configuration, it is know (cfr. [7,8] for further details) that there exist two families of relative equilibria where the configuration is a rhombus. Set $\Gamma_{1}=\Gamma_{2}=1$ and $\Gamma_{3}=\Gamma_{4}=m$, where $m \in(-1,1]$ is a parameter. Place the vortices at $z_{1}=(1,0), z_{2}=(-1,0), z_{3}=(0, y)$ and $z_{4}=(0,-y)$, forming a rhombus with diagonals lying on the coordinate axis. This configuration is a central configuration provided that

$$
y^{2}=\frac{1}{2}\left(\beta \pm \sqrt{\beta^{2}+4 m}\right), \quad \beta=3(1-m) .
$$

The angular velocity is given by

$$
\omega=\frac{m^{2}+4 m+1}{2\left(1+m y^{2}\right)}=\frac{1}{2}+\frac{2 m}{y^{2}+1} .
$$

Taking plus sign in Eq. (1.2) yields a solution for $m \in(-1,1]$ that always has $\omega>0$. We refer to this solution as rhombus $A$. Taking - in Eq. (1.2) yields a solution for $m \in(-1,0)$ having $\omega>0$ for $m \in(-2+\sqrt{3}, 0)$, but $\omega<0$ for $m \in(-1,-2+\sqrt{3})$. We refer to this solution as rhombus $B$. Assuming that $z$ is the relative equilibrium generated by the rhombus central configuration $\xi$. Then

1. if the central configuration is rhombus $A$, then we have

$$
\mathrm{n}_{-}\left(\widehat{A}_{\Gamma}(\xi)\right)= \begin{cases}0 & \text { if } \quad 0<m \leq 1, \\ 3 & \text { if } \quad-2+\sqrt{3}<m<0, \\ 4 & \text { if } \quad-1<m<-2+\sqrt{3} .\end{cases}
$$


2. if the central configuration is rhombus $B$, then we have

$$
\mathrm{n}_{-}\left(\widehat{A}_{\Gamma}(\boldsymbol{\xi})\right)=\left\{\begin{array}{lll}
2 & \text { if } & -2+\sqrt{3}<m<0 \\
4 & \text { if } & m^{*}<m<-2+\sqrt{3} \\
3 & \text { if } \quad-1<m<m^{*}
\end{array}\right.
$$

where $m^{*}$ is the only real root of the cubic $9 m^{3}+3 m^{2}+7 m+5$.

\section{Notation}

At last, let us introduce some notation that we shall use henceforth without further reference. We have already mentioned that I stands for the angular impulse, however, the similar symbol $I_{X}$ or just $I$ will denote the identity operator on a space $X$ and we set for simplicity $I_{k}:=I_{\mathbb{R}^{k}}$ for $k \in \mathbb{N}$. We denote throughout by the symbol $\#^{\top}$ (resp. $\#^{-\top}$ ) the transpose (resp. inverse transpose) of the operator \#.

$\operatorname{Mat}(m, n ; \mathbb{K})$ stands for the space of $m \times n$ matrices in the field $\mathbb{K}$ and if $m=n$ we just use the short-hand notation $\operatorname{Mat}(m ; \mathbb{K}) . \sigma(\#)$ denotes the spectrum of the linear operator \#. We denote throughout by $J$ the standard symplectic matrix $J:=\left[\begin{array}{cc}0 & 1 \\ -1 & 0\end{array}\right]$. $\mathbb{U}$ denotes the unit circle in the complex plane namely the set of all complex numbers of modulus 1 .

If $Z$ is a finite dimensional vector space. We denote by $\mathscr{L}(Z)$ the vector space of all linear operators on $Z$.

\section{The Geometric and Dynamical Framework}

This section essentially focus on describing the geometric and dynamical framework as well as to fix our basic definitions and notation. A similar description already appears in $[8$, Sect. 2] and references therein. However, since the setting is slightly general, we decided to do an effort and to include here for the sake of the reader.

In the Euclidean plane $(V,\langle\cdot, \cdot\rangle)$ equipped with coordinates $z=(p, q)$, we consider the standard symplectic form $\omega$ defined as follows

$$
\Omega(\cdot, \cdot)=\langle J \cdot, \cdot\rangle \quad \text { where } \quad J:=\left[\begin{array}{cc}
0 & 1 \\
-1 & 0
\end{array}\right] .
$$

For $i \in\{1, \ldots, N\}$, let $\Gamma_{i} \in \mathbb{R} \backslash\{0\}$ representing the vortex strength of the $N$-point vortex $z_{i} \in V$. We will assume throughout that the total circulation $\Gamma:=\sum_{i=1}^{N} \Gamma_{i}$ is nonzero. The center of vorticity is then well-defined as $c:=\Gamma^{-1} \sum_{i=1}^{N} \Gamma_{i} z_{i}$. Let $H: V^{N} \rightarrow \mathbb{R}$ be the function defined as follows

$$
H(z)=-\sum_{i<j} \Gamma_{i} \Gamma_{j} \log r_{i j} \quad \text { where } \quad r_{i j}:=\left|z_{i}-z_{j}\right|
$$


for $z=\left(z_{1}, \ldots, z_{N}\right) \in V^{N}$. In what follows we refer to $H$ as the $N$-vortex Hamiltonian function. Denoting by $I_{N}$ the $N \times N$ identity matrix, we define the matrix of circulations as the real $2 N \times 2 N$ matrix given by

$$
M_{\Gamma}:=\left[\begin{array}{ccc}
\Gamma_{1} & & \\
& \ddots & \\
& & \Gamma_{N}
\end{array}\right] \otimes I_{2}=\left[\begin{array}{ccc}
\Gamma_{1} I_{2} & & \\
& \ddots & \\
& & \Gamma_{N} I_{2}
\end{array}\right] \in \operatorname{Mat}(2 N ; \mathbb{R})
$$

and the symplectic matrix $K:=I_{N} \otimes J \in \operatorname{Mat}(2 N ; \mathbb{R})$. Let $\mathbb{F}_{N}(V)$ be the space of all $N$ (colored) points in $V$; in symbols

$$
\mathbb{F}_{N}(V):=\left\{z \in V^{N} \mid i \neq j \Longrightarrow z_{i} \neq z_{j}\right\}=V^{N} \backslash \Delta
$$

Its complement in $V^{N}$ is the collision set

$$
\Delta:=\left\{z \in V^{N} \mid \exists(i, j), i \neq j: z_{i}=z_{j}\right\}=\bigcup_{1 \leq i<j \leq N} \Delta_{i j}
$$

where $\Delta_{i j}:=\left\{z \in V^{N} \mid z_{i}=z_{j}\right\}$. It is immediate to check that the restriction of $H$ to $\mathbb{F}_{N}(V)$ is indeed a smooth function.

\subsection{Central Configurations}

Given two vectors $\boldsymbol{v}, \boldsymbol{w}$ in $V^{N}$, then we let

$$
\langle\boldsymbol{v}, \boldsymbol{w}\rangle_{\Gamma}:=\sum_{i=1}^{N} \Gamma_{i} \boldsymbol{v}_{i} \cdot \boldsymbol{w}_{i}
$$

denote the circulation scalar product of $\boldsymbol{v}$ and $\boldsymbol{w}$, where $\boldsymbol{v}_{i} \cdot \boldsymbol{w}_{i}$ denotes the standard Euclidean product in $V$ of the $i$-th component of $\boldsymbol{v}$ and $\boldsymbol{w}$. We observe that, if the vortex strengths are all positive, then $\langle\cdot, \cdot\rangle_{\Gamma}$ is, actually, an inner product equivalent to the Euclidean one; otherwise, is an indefinite (non-degenerate) scalar product. ${ }^{1} \mathrm{We}$ also notice that $\langle\boldsymbol{v}, \boldsymbol{w}\rangle_{\Gamma}=\boldsymbol{w}^{\top} M_{\Gamma} \boldsymbol{v}$ where $\cdot^{\top}$ denotes the transpose with respect to the Euclidean product. Given $I_{0} \in \mathbb{R}$, we define the pseudo-sphere $\mathbb{S}_{N}(V)$ and we'll refer to as circulation (pseudo)-sphere or circulation sphere for short as

$$
\mathbb{S}:=\mathbb{S}_{N}(V):=\left\{z \in \mathbb{F}_{N}(V) \mid\|z\|_{\Gamma}^{2}=I_{0}\right\}
$$

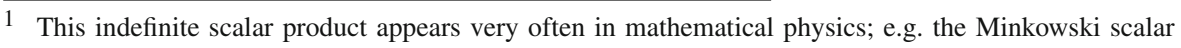
product.
} 
In particular the circulation sphere is equal to the sphere (with respect to the circulation scalar product) in $V^{N}$ with collisions removed; thus $\mathbb{S}_{N}(V)=S_{N}(V) \backslash \Delta$ where

$$
S_{N}(V):=\left\{z \in V^{N} \mid\|z\|_{\Gamma}^{2}=I_{0}\right\}
$$

Remark 2.1 It is worth noticing that if vortex strengths are all positive, then the pseudosphere is in general an ellipsoid (thus, topologically a sphere) and if are all equal it reduces to the round sphere. In the general case, however, it is a (non-compact) quadric. (Cfr. [8, Sect. 2.2] for an exhaustive discussion in the positive definite case).

A central configuration for the the $N$-vortex problem is a (non-collision) configuration $\xi \in \mathbb{F}_{N}(V)$ with the property that exists $\omega \in \mathbb{R}$ such that

$$
\nabla H(\xi)+\omega \nabla I(\xi)=\nabla H(\xi)+\omega M_{\Gamma}(\xi)=0 .
$$

Let $C: V^{N} \rightarrow V^{N}$ be the isometry defined by $C(\xi)=\xi^{\prime}$, with

$$
\xi_{j}^{\prime}=\xi_{j}-2 c
$$

for each $j=1, \ldots, N$. It is easy to check that $\boldsymbol{C}$ is an isometry with respect to the $\langle\cdot, \cdot\rangle_{\Gamma}$. In fact, it holds

$$
\begin{aligned}
\left\|\boldsymbol{\xi}^{\prime}\right\|_{\Gamma}^{2} & =\sum_{i=1}^{N}\left\|\boldsymbol{\xi}_{i}-2 \boldsymbol{c}\right\|_{\Gamma}^{2}=\sum_{i=1}^{N}\left\langle\Gamma_{i} \boldsymbol{\xi}_{i}, \boldsymbol{\xi}_{i}\right\rangle_{\Gamma}-4 \sum_{i=1}^{N}\left\langle\Gamma_{i} \boldsymbol{\xi}_{i}, \boldsymbol{c}\right\rangle_{\Gamma}+4 \sum_{i=1}^{N} \Gamma_{i}\|\boldsymbol{c}\|_{\Gamma}^{2} \\
& =\|\boldsymbol{\xi}\|_{\Gamma}^{2}-4\|\boldsymbol{c}\|_{\Gamma}^{2} \Gamma+4\|\boldsymbol{c}\|_{\Gamma}^{2} \Gamma=\|\boldsymbol{\xi}\|_{\Gamma}^{2} .
\end{aligned}
$$

We observe that $H(\boldsymbol{C} \xi)=H(\xi)$ and by the computation performed in Eq. (2.3), we conclude immediately that $I\left(\xi^{\prime}\right)=I(\xi)$. By these two fact readily follows that if $\xi$ is a central configuration then also $\boldsymbol{C} \boldsymbol{\xi}$ is a central configuration. Now, by using Eq. (2.2), we can conclude that $\boldsymbol{C} \boldsymbol{\xi}=\boldsymbol{\xi}$ and hence $\boldsymbol{\xi}=0$. Thus, if $\boldsymbol{\xi}$ is a central configuration, then its center of vorticity $c=0$. As consequence of this discussion and without leading in generalities in the sequel we'll restrict to the reduced phase space which is the $2(N-1)$-dimensional subspace of $V^{N}$ defined by

$$
\mathcal{X}:=\left\{z=\left(z_{1}, \ldots, z_{N}\right) \in V^{N} \mid \sum_{i=1}^{N} \Gamma_{i} z_{i}=0\right\} .
$$

By using once again Eq. (2.2) it follows in fact, that a central configuration can be seen as a critical point of the Hamiltonian function restricted to a level surface of the angular impulse in which $\omega$ acts as a Lagrangian multiplier. Note that, if $\bar{z}$ is a central configuration, so is $\lambda \bar{z}$ for any scalar $\lambda$. In this case, the parameter $\omega$ must be scaled by a factor $1 / \lambda^{2}$. 
We observe that by the invariance property of the Hamiltonian function as well as of the angular-impulse, we get that central configurations are not isolated and appears in a continuous family. To eliminate such a degeneracy, it is customary to fix a scaling (e.g. $I=I_{0}$ ) and to identify central configurations that are identical under rotations.

Remark 2.2 It is also worth noticing that the linear stability properties of such rigid motions are not affected by such rotation.

We also define the following sets

$$
\mathbb{S}_{N}^{c}:=\mathbb{S}_{N} \cap \mathcal{X} \quad \text { and } \quad S_{N}^{c}:=S_{N} \cap \mathcal{X}
$$

By the above discussion, in particular we get that if $z$ is a central configuration then $z \in$ $\mathcal{X}$. However, in principle, a critical point of the restriction of $\left.H\right|_{\mathbb{S}_{N}^{c}}$ is not necessarily a critical point of $\left.H\right|_{\mathbb{S}_{N}}$. However since the Hamiltonian function is $\boldsymbol{C}$-invariant and being $\mathcal{X}$ the space fixed by the action of the (compact Lie) orthogonal group of $V^{N}$, it follows that any critical point of the restriction of $\left.H\right|_{\mathbb{S}_{N}^{c}}$ is indeed a critical point of $\left.H\right|_{\mathbb{S}_{N}}$ (cf. [10], for further details). However, as already observed critical points of $\left.H\right|_{\mathbb{S}_{N}^{c}}$ are not isolated. In fact, if $z_{0}$ is a critical point of $\left.H\right|_{\mathbb{S}_{N}^{c}}$, then $e^{\vartheta K} z_{0}$ is, for every $\vartheta$. In order to eliminate this further degeneracy, we consider the orbit spaces

$$
\widehat{\mathbb{S}}_{N}^{c}:=\mathbb{S}_{N}^{c} / \mathrm{SO}(2) \quad \text { and } \quad \widehat{S}_{N}^{c}:=S_{N}^{c} / \mathrm{SO}(2)
$$

and we'll refer to the shape sphere without collision and the shape sphere respectively. It is worth noticing that both are the orbit space of the circle action on the spheres $\mathbb{S}_{N}^{c}$ and $S_{N}^{c}$, respectively. In what follows, we'll refer to a central configuration as the critical point of $H_{\widehat{S}_{N}^{c}}$ in order to distinguish from critical points of $H_{\mathbb{S}_{N}^{c}}$ usually called relative equilibria.

Remark 2.3 As already observed by author in [8, Sect. 2.2] since $\mathrm{SO}(2) \equiv S^{1}$ acts freely on $\mathbb{S}_{N}^{c}$, we get that $\widehat{\mathbb{S}}_{N}^{c}$ is also a manifold. For the case of three vortices (having positive circulations), it is actually diffeomorphic to the 2-dimensional sphere minus three points corresponding to the binary collisions (triple collision being a priori eliminated by the normalization condition induced by restricting to the level set of the angular impulse).

\subsection{Relative Equilibria}

A system of $N$ point vortices (in the plane) with vortex strength $\Gamma_{i} \neq 0$ and positions $z_{i} \in V$ evolves according to the phase flow induced by the following Hamiltonian system

$$
\Gamma_{i} \dot{z}_{i}=J \nabla_{i} H(z(t))=J \sum_{j \neq i}^{N} \frac{\Gamma_{i} \Gamma_{j}}{r_{i j}^{2}}\left(z_{j}-z_{i}\right), \quad i \in\{1, \ldots, n\}
$$

where the Hamiltonian function $H$ is defined in Eq. (2.1), and $\nabla_{i}$ denotes the twodimensional partial gradient with respect to $z_{i}$. We will assume throughout that 
(H) the total circulation $\Gamma=\sum_{i=1}^{N} \Gamma_{i}$ is nonzero and that the center of vorticity $c=0$.

In short-hand notation Eq. (2.4), could be rewritten in the following form

$$
M_{\Gamma} \dot{z}(t)=K \nabla H(z(t)), \quad t \in[0,2 \pi]
$$

where $K$ is the real $2 N \times 2 N$ matrix given by

$$
K:=I_{N} \otimes J=\left[\begin{array}{lll}
J & & \\
& \ddots & \\
& & J
\end{array}\right] \in \operatorname{Mat}(2 N ; \mathbb{R}) .
$$

A special class (maybe the easiest) of periodic solutions for this problem is given by the rigid motions of the system around its center of mass. Such a motions are termed relative equilibria. More precisely we introduce the following definition.

Definition 2.4 We term relative equilibrium (RE, for short) any $T:=2 \pi /|\omega|$ periodic solution of Eq. (2.5), namely

$$
z(t):=e^{-\omega K t} \xi, \quad \text { where } \omega \in \mathbb{R} \backslash\{0\}, \quad t \in[0, T] \text { and } \boldsymbol{\xi} \in \mathbb{F}_{N}(V) .
$$

Remark 2.5 By this definition, as already observed, it follows that a relative equilibrium is a periodic solution in which each point vortex uniformly rotates with angular velocity $\omega \neq 0$ around (its common center of vorticity represented by) the origin.

By a direct computation and by using Eq. (2.6), it follows that the central configuration $\xi$ generating a relative equilibrium satisfy the following equation

$$
-\omega \Gamma_{i} \xi_{i}=\nabla_{i} H(\xi)=\sum_{j \neq i}^{N} \frac{\Gamma_{i} \Gamma_{j}}{r_{i j}^{2}(0)}\left(\xi_{j}-\xi_{i}\right), \quad \text { for each } i \in\{1, \ldots, n\}
$$

Otherwise said, for every $i \in\{1, \ldots, n\}, \nabla_{i} H(\xi)+\omega \Gamma_{i} \xi=0$ which is equivalent to claim that $\xi$ is a solution of Eq. (2.2) hence a central configuration. Thus in a properly rotating frame a relative equilibrium is nothing but a central configuration.

The following result points out some crucial properties of the Hamiltonian function $H$ that will be useful later on and we refer the interested reader to [7, Lemma 2.3] for the proof.

Lemma 2.6 The Hamiltonian H has the following three properties:

(i) $\nabla H(z) \cdot z=-L$,

(ii) $\nabla H(z) \cdot(K z)=0$,

(iii) $D^{2} H(z) K=-K D^{2} H(z)$.

Remark 2.7 As we will see later on, property (iii) plays a crucial role in the investigation of the linear stability for relative equilibria. For all of the same sign vorticity strengths, 
such a condition reduces the problem to the investigation of the spectrum to a $2 \times$ 2 symmetric matrix or equally well a complete factorization of the characteristic polynomial into even quadratic factors.

This property doesn't hold for relative equilibria of the $N$-body problem and in fact a challenging longstanding still open problem is to establish a precise relation between the dynamical properties of the relative equilibria and the spectral properties of central configurations originating them.

Differentiating with respect to $z$ the equality appearing at first item in Lemma 2.6, we get

$$
\begin{aligned}
\left\langle D^{2} H(\boldsymbol{\xi})[\boldsymbol{u}], z\right\rangle+\langle\nabla H(\xi), \boldsymbol{u}\rangle & =\left\langle D^{2} H(\boldsymbol{\xi})[z], \boldsymbol{u}\right\rangle+\langle\nabla H(\boldsymbol{\xi}), \boldsymbol{u}\rangle=0, \quad \forall \boldsymbol{u} \in T_{\boldsymbol{\xi}} \widehat{\mathbb{S}}_{N}^{\boldsymbol{c}} \\
& \Rightarrow D^{2} H(\boldsymbol{\xi})[\boldsymbol{z}]+\nabla H(\boldsymbol{\xi})=0 .
\end{aligned}
$$

Since $\boldsymbol{\xi}$ is a central configuration and by using once again Eq. (2.2), we immediately get $\nabla H(\xi)=-\omega M_{\Gamma} \xi$ and by summing up we get the equality

$$
M_{\Gamma}^{-1} D^{2} H(\xi) \xi=\omega \xi
$$

Equation (2.7) together with property (iii) Lemma 2.6 shows that $B(\xi) K \xi=0$. The equation of motions given in Eq. (2.5), in a uniformly rotating frame with angular velocity $\omega$ reduces to

$$
M_{\Gamma} \dot{\boldsymbol{w}}(t)=K(\nabla H(\boldsymbol{w}(t))+\omega M \boldsymbol{w}(t)), \quad t \in\left[0, \frac{2 \pi}{|\omega|}\right]
$$

In fact, let $\boldsymbol{w}(t):=e^{\omega K t} \boldsymbol{z}(t)$; thus by a direct computation, we get

$$
M_{\Gamma} \dot{\boldsymbol{w}}(t)=\omega K M_{\Gamma} e^{\omega K t} \boldsymbol{z}(t)+M_{\Gamma} e^{\omega K t} \nabla H(z(t))=K\left[\nabla H(\boldsymbol{w}(t))+\omega M_{\Gamma} \boldsymbol{w}(t)\right]
$$

where the commutativity properties of $M_{\Gamma}$ with respect to $K$ and $e^{K}$ were tacitly used. In particular, a rest point of the Hamiltonian vector field appearing in Eq. (2.8) is a relative equilibrium, as expected.

Remark 2.8 We observe that if the circulations have mixed sign then $\omega$ could be of any sign (meaning that the vortices can rotate clockwise or counterclockwise with respect to the center of vorticity). In fact, by taking the scalar product with respect to $\xi$ in Eq. (2.2) as well as invoking the first claim in Lemma 2.6, we get that

$$
L=\omega\langle\nabla I(\boldsymbol{\xi}), \boldsymbol{\xi}\rangle=2 \omega I(\boldsymbol{\xi}) \Rightarrow \omega=L /(2 I(\boldsymbol{\xi}))
$$

where the last equality directly follows by using the Euler theorem on positively homogeneous functions after observing that $I$ is homogeneous of degree 2 . Now, the claim follows by observing that a priori $L$ could be of either positive or negative. 
Remark 2.9 In a more geometrical way the Hamilton equations in the uniformly rotating frame are nothing but the Hamilton equation on the cotangent bundle $T^{*} S_{N}^{c}$ with the symplectic form induced by the standard symplectic form whose Hamiltonian vectorfield (i.e. the symplectic gradient) is defined by

$$
X_{H}(\boldsymbol{w}):=K\left[\nabla H(\boldsymbol{w})+\omega M_{\Gamma} \boldsymbol{w}\right]
$$

The variational equation associated to the Hamiltonian system given in Eq. (2.8) is

$$
\left.M_{\Gamma} \dot{\boldsymbol{\xi}}(t)=K\left[D^{2} H(\boldsymbol{w}(t))+\omega M\right] \boldsymbol{\xi}(t)\right), \quad t \in\left[0, \frac{2 \pi}{|\omega|}\right]
$$

In particular if $\boldsymbol{w}(t)=e^{\omega K t} \boldsymbol{\xi}$ is a relative equilibrium solution at the central configuration $\xi$, and the admissible variations belongs to the tangent along the fibers of the principal $S^{1}$-bundle, then

$$
D^{2} H(\boldsymbol{w}(t))=D^{2} H(\boldsymbol{\xi}) .
$$

In fact, since $H$ is invariant under rotation, it follows that $H(z(t))=H(\xi)$. Now, by differentiating twice this last equality for $\boldsymbol{w}(t)=e^{\omega K t} \boldsymbol{\xi}$ (here the admissible variations are of the form $e^{\omega K t} \boldsymbol{u}$ for $\boldsymbol{u} \in T \mathbb{S}_{N}^{c}$ ), we get

$$
e^{-\omega K t} D^{2} H(\boldsymbol{w}(t)) e^{\omega K t}=D^{2} H\left(z_{0}\right)
$$

Inserting the expression given in Eq. (2.10) into Eq. (2.8) and setting $\boldsymbol{\eta}=e^{\omega K t} \boldsymbol{\xi}$, we get

$$
\dot{\boldsymbol{\eta}}(t)=K\left[M_{\Gamma}^{-1} D^{2} H(\xi)+\omega I\right] \boldsymbol{\eta}(t) .
$$

Following Roberts [7] we introduce the following definition.

Definition 2.10 The matrix

$$
B(\xi):=K A_{\Gamma}(\xi) \quad \text { where } \quad A_{\Gamma}(\xi):=\left[M_{\Gamma}^{-1} D^{2} H(\xi)+\omega I\right]
$$

is termed the stability matrix of the relative equilibrium $z$ generated by $\xi$. Borrowing the definition introduced by author in [8, Sect. 2.2], we sometimes, refer to $A_{\Gamma}(\xi)$ as modified Hessian.

Notation 2.11 When no confusion may occur, in shorthand notation we denote by $B$ (resp. $A_{\Gamma}$ ) the matrices $B(\xi)($ resp. $A(\xi))$.

Definition 2.12 Let $\left(\mathbb{R}^{k}, N\right)$ be a (maybe indefinite) non-degenerate scalar product space on the real vector space $\mathbb{R}^{k}$ and let $G \in \operatorname{Mat}(k, \mathbb{R})$. The matrix $G$ is termed a $N$-symmetric matrix if

$$
N G=G^{\top} N
$$


where ${ }^{\top}$ denotes the transpose with the respect to the Euclidean product. The matrix $R \in \operatorname{Sp}(2 n, \Omega)$ is termed $N$-Hamiltonian, if

$$
R^{\top} P N+N P R=0
$$

where $P$ represents the symplectic form $\Omega$ with respect to the Euclidean product.

Remark 2.13 It is worth noticing that if $N=I$ then the definitions of $N$-symmetric (resp. $N$-Hamiltonian) matrix, reduces to the standard definition of symmetric (resp. Hamiltonian) matrix with respect to the Euclidean scalar product (resp. canonical symplectic structure).

By a direct calculation follows that $M_{\Gamma}^{-1} D^{2} H(\xi)(\xi)$ is a $M_{\Gamma}$-symmetric matrix (whatever the sign of each circulation is) and $B(\xi)$ is $M_{\Gamma}$-Hamiltonian, meaning that

$$
B^{\top}(\xi) K M_{\Gamma}+M_{\Gamma} K B(\xi)=0
$$

This last claim directly follows by Eq. (2.7). Otherwise stated, the matrix $B(\xi)$ is Hamiltonian with respect to the vortex symplectic form defined by

$$
\omega_{\Gamma}(\cdot, \cdot)=\langle K \cdot, \cdot\rangle_{\Gamma}=\left\langle M_{\Gamma} K \cdot, \cdot\right\rangle .
$$

Remark 2.14 We pause the exposition by introducing the following remark that explain why the mixed sign circulations case is really completely different from the constant sign circulations. It is well-known that the product of two symmetric matrices is symmetric as soon as the two matrices commute. Thus, in general, the matrix $M_{\Gamma}^{-1} D^{2} H(\xi)(\xi)$ whatever $\xi$ is, and no matter how the signs of $\Gamma_{i}$ arem not be symmetric. Clearly, if all circulations are equal then $M_{\Gamma}$ is just a multiple of the identity and, of course, $M_{\Gamma}^{-1} D^{2} H(\xi)$ is symmetric. However, if the circulations strengths $\Gamma_{i}$ are all positive, then the matrix $M_{\Gamma}$ is positive definite and in particular its spectrum is real. For mixed signs circulation strengths, however, $M_{\Gamma}$-is (nondegenerate) but indefinite and its spectrum [11, Theorem 5.1.1, p.74] is not necessarily real, anymore and this fact is responsible among others of some technicalities as well as a deep change in the dynamics of the problem.

We conclude this section by showing a nice and important block matrix structure of the Hessian matrix $D^{2} H(\xi)$. This property comes from item (iii) in Lemma 2.6. Let $\xi$ be a central configuration; thus $\boldsymbol{\xi}=\left(\boldsymbol{\xi}_{1}, \boldsymbol{\xi}_{2}, \ldots, \boldsymbol{\xi}_{n}\right) \in \mathbb{R}^{2 n}$ and let $\boldsymbol{\xi}_{i j}=\left(\boldsymbol{\xi}_{i}-\boldsymbol{\xi}_{j}\right) / r_{i j}$. A direct computation shows that

$$
D^{2} H(\xi)=\left[\begin{array}{cccc}
A_{11} & A_{12} & \cdots & A_{1 n} \\
\vdots & & \vdots \\
A_{n 1} & A_{n 2} & \cdots & A_{n n}
\end{array}\right]
$$


where $A_{i j}$ is the $2 \times 2$ symmetric matrix

$$
\begin{cases}A_{i j}:=\frac{\Gamma_{i} \Gamma_{j}}{r_{i j}^{2}}\left[I-2 \xi_{i j} \xi_{i j}^{\top}\right] \quad & \text { if } i \neq j \\ A_{i i}:=-\sum_{j \neq i} A_{i j} & \text { otherwise. }\end{cases}
$$

Note that $A_{i j}=A_{j i}$ and, for $i \neq j$

$$
A_{i j}=\frac{\Gamma_{i} \Gamma_{j}}{r_{i j}^{4}}\left[\begin{array}{cc}
\left(y_{i}-y_{j}\right)^{2}-\left(x_{i}-x_{j}\right)^{2} & -2\left(x_{i}-x_{j}\right)\left(y_{i}-y_{j}\right) \\
-2\left(x_{i}-x_{j}\right)\left(y_{i}-y_{j}\right) & \left(x_{i}-x_{j}\right)^{2}-\left(y_{i}-y_{j}\right)^{2}
\end{array}\right]
$$

where $\xi=(x, y)$. The fact that $J$ commutes with each $A_{i j}$ gives another proof of the fact that $D^{2} H(\xi)$ and $K$ anti-commute.

\section{Lemma 2.15 The following facts hold:}

1. $s=[1,0,1,0, \ldots, 1,0], K s \in \operatorname{ker} D^{2} H(\xi)$,

2. For every relative equilibrium $z$ generated by the central configuration $\xi$, we have that $K \xi \in \operatorname{ker} B$.

Proof From the conservation of the center of vorticity, by using Eq. (2.11) and by a straightforward calculation we get that

$$
\sum_{i=1}^{n} A_{j i}\left(\begin{array}{l}
1 \\
0
\end{array}\right)=\sum_{i \neq j} A_{j i}\left(\begin{array}{l}
1 \\
0
\end{array}\right)+A_{j j}\left(\begin{array}{l}
1 \\
0
\end{array}\right)=\sum_{i \neq j} A_{j i}\left(\begin{array}{l}
1 \\
0
\end{array}\right)-\sum_{i \neq j} A_{j i}\left(\begin{array}{l}
1 \\
0
\end{array}\right)=0
$$

This implies that

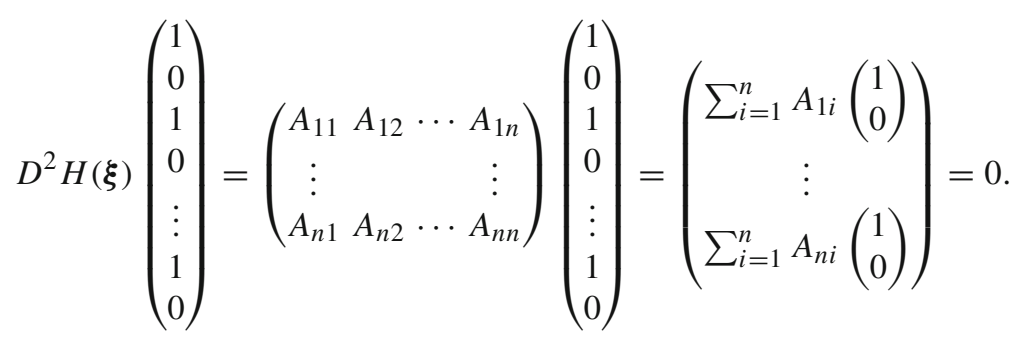

Thus $s \in \operatorname{ker} D^{2} H(\xi)$.

Furthermore, by the last claim in Lemma 2.6, we have $D^{2} H(\xi) K \boldsymbol{s}=-K D^{2} H(\boldsymbol{\xi}) \boldsymbol{s}$ $=0$ which implies that also $K s \in \operatorname{ker} D^{2} H(\xi)$.

The proof of the second claim directly follows by Eq. (2.7) together with property (iii) Lemma 2.6. In fact, by a direct computation we get

$$
\begin{aligned}
B \boldsymbol{\xi} & =K\left[M_{\Gamma}^{-1} D^{2} H(\boldsymbol{\xi}) \boldsymbol{\xi}+\omega \boldsymbol{\xi}\right]=K[\omega \boldsymbol{\xi}+\omega \boldsymbol{\xi}]=2 \omega K \boldsymbol{\xi} \\
B K \boldsymbol{\xi} & =K\left[M_{\Gamma}^{-1} D^{2} H(\boldsymbol{\xi}) K \boldsymbol{\xi}+\omega K \boldsymbol{\xi}\right]=K\left[-K M_{\Gamma}^{-1} D^{2} H(\boldsymbol{\xi}) \boldsymbol{\xi}+\omega K \boldsymbol{\xi}\right]
\end{aligned}
$$




$$
=K[-\omega K \xi+\omega K \xi]=0 .
$$

This concludes the proof.

By the first claim in Lemma 2.15 it then follows that the restriction of the stability matrix to the spectrum of $B$ is precisely $\{ \pm \omega i\}$. For a given relative equilibrium $z$ with corresponding central configuration $\boldsymbol{\xi}$, let $W=\operatorname{span}\{\xi, K \xi\}$. As already proved in Lemma 2.15 this is an invariant subspace for $B$ and the restriction of $B$ to $W$ is given by

$$
\left.B\right|_{W}=\left[\begin{array}{cc}
0 & 0 \\
2 \omega & 0
\end{array}\right]
$$

Notation 2.16 In what follows, we denote by $W^{\perp} \subset V^{N}$ the $M_{\Gamma^{-}}$orthogonal complement of $W$, that is,

$$
W^{\perp}=\left\{\boldsymbol{w} \in \mathbb{R}^{2 n}:\langle\boldsymbol{w}, \boldsymbol{v}\rangle_{\Gamma}=\boldsymbol{w}^{\top} M_{\Gamma} \boldsymbol{v}=0, \forall \boldsymbol{v} \in W\right\}
$$

Lemma 2.17 The vector space $W^{\perp}$ has dimension $2 n-2$ and is invariant under $B$. If $L \neq 0$, then $W \cap W^{\perp}=\{0\}$.

Proof For the proof of this result, we refer the interested reader to [7, Lemma 2.6].

As long as $L \neq 0$, Lemma 2.17 allows us to define the linear stability with respect to the $M_{\Gamma}$-orthogonal complement of the subspace $W$. Thus, a relative equilibrium is spectrally (resp. linearly) stable if the restriction of the matrix $B$ onto $W^{\perp}$ is spectrally (resp. linearly) stable according to Definition 1.1. However instead of working on a reduced phase space (eliminating the rotational symmetry), it is more convenient to work on the full phase space we investigate the linear stability properties of the orbits in the full space. However, in this case, by the invariance properties of $H$, it readily follows that 4 is the minimal possible nullity (or kernel dimension) of the corresponding linear differential operator.

\section{Spectral Properties and Canonical Forms of the Stability Matrix}

This section is devoted to collect several linear algebraic results on the spectrum of the stability matrix $B$ that we will need in order to prove our main result. In fact, as already observed, the eigenvalues of $B$ determine the spectral and linear stability of the corresponding periodic solution.

\subsection{Spectral Properties of the Stability Matrix}

The aim of this subsection is to study the relation intertwining the spectrum of the stability matrix $B$ and the spectrum of $M_{\Gamma}^{-1} D^{2} H(\xi)$ (and hence of $\widehat{A}$ ). The first result, that we recall here for the sake of the reader, was proved by Roberts in [7]. 
Lemma 3.1 The characteristic polynomials of $M_{\Gamma}^{-1} D^{2} H(\xi)$ and $D^{2} H(\xi)$ are even.

Moreover, for each on of the above matrices, $v$ is an eigenvector with eigenvalue $\mu$ if and only if $K \boldsymbol{v}$ is an eigenvector with eigenvalue $-\mu$.

Proof The proof of this result follows by a direct computation. (Cf. [7, Lemma 2.4], for further details).

The following result relates the spectrum of $B$ with the spectrum of the matrix $M_{\Gamma}^{-1} D^{2} H(\xi)$ and will be a key ingredient for the stability analysis.

Lemma 3.2 Under the above notation, $\lambda \in \sigma(B)$ if and only if $\mu_{ \pm}:= \pm \sqrt{\lambda^{2}+\omega^{2}} \in$ $\sigma\left(M_{\Gamma}^{-1} D^{2} H(\xi)\right)$, where $\sqrt{\cdot}$ denotes the the square root of the (maybe real negative or complex number $\left.\lambda^{2}+\omega^{2}\right)$. More precisely, the following facts hold:

1. $\lambda \in \sigma(B) \cap i \mathbb{R})$ iff $\mu \in \sigma\left(M_{\Gamma}^{-1} D^{2} H(\xi)\right) \cap i \mathbb{R}$ or $\mu \in \sigma\left(M_{\Gamma}^{-1} D^{2} H(\xi)\right) \cap \mathbb{R}$ and $|\mu| \in[-|\omega|,|\omega|]$.

2. $\lambda \in \sigma(B) \cap \mathbb{R})$ iff $\mu \in \sigma\left(M_{\Gamma}^{-1} D^{2} H(\xi)\right) \cap \mathbb{R}$ and $|\mu| \geq|\omega|$.

3. $\lambda \in \sigma(B) \cap \mathbb{C} \backslash(\mathbb{R} \cup i \mathbb{R})$ iff $\mu \in \sigma\left(M_{\Gamma}^{-1} D^{2} H(\xi)\right) \cap \mathbb{C} \backslash(\mathbb{R} \cup i \mathbb{R})$.

Proof Since $K^{2}=-I$ and $K M_{\Gamma}^{-1} D^{2} H(\xi)=-M_{\Gamma}^{-1} D^{2} H(\xi) K$ (as direct follows by applying Lemma 2.6), then by a direct computation, we get:

$$
\begin{aligned}
B-\lambda^{2} I & =(B-\lambda)(B+\lambda)=\left[K\left(M_{\Gamma}^{-1} D^{2} H(\xi)+\omega I\right)-\lambda I\right]\left[K\left(M_{\Gamma}^{-1} D^{2} H(\xi)+\omega I\right)+\lambda I\right] \\
& =K\left(M_{\Gamma}^{-1} D^{2} H(\xi)+\omega I\right) K\left(M_{\Gamma}^{-1} D^{2} H(\xi)+\omega I\right)-\lambda^{2} I=\left(K M_{\Gamma}^{-1} D^{2} H(\xi)+K \omega I\right)^{2}-\lambda^{2} I \\
& =M_{\Gamma}^{-1} D^{2} H(\xi)^{2}+K M_{\Gamma}^{-1} D^{2} H(\xi) K \omega+K \omega K M_{\Gamma}^{-1} D^{2} H(\xi)-\lambda^{2} I-\omega^{2} I \\
& =M_{\Gamma}^{-1} D^{2} H(\xi)^{2}+M_{\Gamma}^{-1} D^{2} H(\xi) \omega-\omega M_{\Gamma}^{-1} D^{2} H(\xi)-\lambda^{2} I-\omega^{2} I \\
& =M_{\Gamma}^{-1} D^{2} H(\xi)^{2}-\left(\lambda^{2}+\omega^{2}\right) I .
\end{aligned}
$$

By the calculation performed in Eq. (3.1) we get that

$$
\begin{aligned}
0 & =\operatorname{det}\left(B-\lambda^{2} I\right) \quad \text { iff } \quad \operatorname{det}\left[M_{\Gamma}^{-1} D^{2} H(\xi)^{2}-\left(\lambda^{2}+\omega^{2}\right) I\right] \\
& =\quad \operatorname{det}\left[M_{\Gamma}^{-1} D^{2} H(\xi)^{2}-\mu^{2} I\right]=0
\end{aligned}
$$

where $\mu^{2}:=\lambda^{2}+\omega^{2}$.

In order to prove the first claim, we start to observe that if $\lambda \in \sigma(B) \cap i \mathbb{R}$ then $\lambda^{2}=\mu^{2}-\omega^{2}<0$. By this last inequality we can conclude that either $\mu^{2}<0$ or $0<\mu^{2} \leq \omega^{2}$ or which is equivalent to state that $\mu \in \sigma\left(M_{\Gamma}^{-1} D^{2} H(\xi)\right) \cap i \mathbb{R}$ or $\mu \in$ $\sigma\left(M_{\Gamma}^{-1} D^{2} H(\xi)\right) \cap \mathbb{R}$ and $|\mu| \in[-|\omega|,|\omega|]$. Viceversa, if $\mu \in \sigma\left(M_{\Gamma}^{-1} D^{2} H(\xi)\right) \cap i \mathbb{R}$, in particular $\mu^{2}<0$. Being $\mu^{2}=\lambda^{2}+\omega^{2}<0$ and $\omega^{2}>0$ (being $\omega \in \mathbb{R}$ ) this implies that $\lambda^{2}<0$. Thus $\lambda \in i \mathbb{R}$ and by Eq. (3.1) $\lambda \in \sigma\left(M_{\Gamma}^{-1} D^{2} H(\xi)\right)$. This conclude the proof of the first item. The proof of items 2 and 3 can be proved by arguing precisely as above and we leave the proof to the interested reader. 


\subsection{Canonical Forms and Invariant Splitting of the Phase Space}

This subsection is devoted to study the relation between the invariant subspaces of $B$ (which are crucial for reducing the operator $B$ and the generalized eigenspaces of $A_{\Gamma}$ ). Lemma 3.3 that we state below for the sake of the reader, was proved in [7, Lemma 2.5].

Lemma 3.3 Let $p(\lambda):=\operatorname{det}(B-\lambda I)$ be the characteristic polynomial of the stability matrix $B$.

(a) Suppose that $\boldsymbol{v}$ is a real eigenvector of $M_{\Gamma}^{-1} D^{2} H(\xi)$ corresponding to the eigenvalue $\mu$. Then the span of the two vectors $\{\boldsymbol{v}, K \boldsymbol{v}\}$ is a real invariant subspace of $B$ and the restriction of $B$ to this subspace is given by

$$
\left[\begin{array}{cc}
0 & \mu-\omega \\
\mu+\omega & 0
\end{array}\right] .
$$

Consequently, $p(\lambda)$ has a quadratic factor of the form $\lambda^{2}+\omega^{2}-\mu^{2}$.

(b) Suppose that $\boldsymbol{v}=\boldsymbol{v}_{1}+i \boldsymbol{v}_{2}$ is a complex eigenvector of $M_{\Gamma}^{-1} D^{2} H(\boldsymbol{\xi})$ corresponding to the complex eigenvalue $\mu=\alpha+i \beta$. Then the span of the four vectors $\left\{\boldsymbol{v}_{1}, \boldsymbol{v}_{2}, K \boldsymbol{v}_{1}, K \boldsymbol{v}_{2}\right\}$ is a real invariant subspace of $B$ and the restriction of $B$ to this subspace is given by

$$
\left[\begin{array}{cccc}
0 & 0 & \alpha-\omega & \beta \\
0 & 0 & -\beta & \alpha-\omega \\
\alpha+\omega & \beta & 0 & 0 \\
-\beta & \alpha+\omega & 0 & 0
\end{array}\right]
$$

Consequently, $p(\lambda)$ has a quartic factor of the form $\left(\lambda^{2}+\omega^{2}-\mu^{2}\right)\left(\lambda^{2}+\omega^{2}-\bar{\mu}^{2}\right)$.

Proof The proof of this result follows by a direct computation by using Lemma 3.1. (Cf. [7, Lemma 2.5]).

In the case of mixed signs circulations, the matrix $M_{\Gamma}^{-1} D^{2} H(\xi)$ is $M_{\Gamma}$-symmetric with respect to an indefinite scalar product and this, among others, in particular implies that the spectrum is not real and $M_{\Gamma}^{-1} D^{2} H(\xi)$ and hence $A_{\Gamma}$ are not semi-simple.

Remark 3.4 In the case of positive circulations the symmetry property of $M_{\Gamma}^{-1} D^{2} H(\xi)$ has already been observed in [8, Sect. 2.2].

In order to decompose the full space into $B$ invariant subspaces it is then crucial to understand in which manner Lemma 3.3 can be carried over in this more general situation we are dealing with. This is essentially the content of Lemma 3.5 and Proposition 3.7, below.

Lemma 3.5 Let $\left\{\boldsymbol{v}_{i}\right\}_{i=0}^{l}$ be a Jordan chain of $A_{\Gamma}$ with eigenvalue $v$, namely

$$
\left\{\begin{array}{l}
A_{\Gamma} \boldsymbol{v}_{i+1}=v \boldsymbol{v}_{i+1}+\boldsymbol{v}_{i} \\
\boldsymbol{v}_{0}=0
\end{array}\right.
$$


Then, the set $\left\{K \boldsymbol{v}_{i}\right\}_{i=0}^{l}$ is a Jordan chain of $A_{\Gamma}$ with eigenvalue $2 \omega-v$; thus

$$
\left\{\begin{array}{l}
A_{\Gamma} K \boldsymbol{v}_{i+1}=(2 \omega-v) K \boldsymbol{v}_{i+1}-K \boldsymbol{v}_{i} \\
\boldsymbol{v}_{0}=0
\end{array}\right.
$$

Proof Since $K M_{\Gamma}^{-1} D^{2} H(\xi)=-M_{\Gamma}^{-1} D^{2} H(\xi) K$ (as directly follows by the third item in Lemma 2.6), then by a direct computation, we get

$$
\begin{aligned}
{\left[M_{\Gamma}^{-1} D^{2} H(\boldsymbol{\xi})+\omega I\right] K \boldsymbol{v}_{i+1} } & =K\left(-M_{\Gamma}^{-1} D^{2} H(\boldsymbol{\xi})+\omega I\right) \boldsymbol{v}_{i+1} \\
& =K\left(-M_{\Gamma}^{-1} D^{2} H(\boldsymbol{\xi})-\omega I+2 \omega I\right) \boldsymbol{v}_{i+1} \\
& =-K A_{\Gamma} \boldsymbol{v}_{i+1}+2 \omega K \boldsymbol{v}_{i+1} \\
& =(2 \omega-v) K \boldsymbol{v}_{i+1}-K \boldsymbol{v}_{i} .
\end{aligned}
$$

This concludes the proof.

Remark 3.6 Lemma 3.5 provides a constructive way to reduce the operator $B$ (by decomposing the whole space into $B$-invariant subspaces).

Similar relation between eigenvectors of $A_{\Gamma}$ relative to the eigenvalues $v$ and $2 \omega-v$ already appeared in [8, Lemma 3.3].

Proposition 3.7 Let $\left\{\boldsymbol{v}_{i}\right\}_{i=0}^{l}$ be a Jordan chain of $A_{\Gamma}$ with eigenvalue $v$. Then the span of $\left\{\boldsymbol{v}_{i}, K \boldsymbol{v}_{i}\right\}_{i=1}^{l}$ is an invariant space for $B$.

Proof Since $M_{\Gamma}^{-1} D^{2} H(\xi)$ and $K$ anti-commutes, namely $K M_{\Gamma}^{-1} D^{2} H(\xi)=$ $-M_{\Gamma}^{-1} D^{2} H(\xi) K$, by a direct computation of $B \boldsymbol{v}_{i+1}$ and $B K \boldsymbol{v}_{i+1}$ and by taking advantage of Lemma 3.5, we immediately get

$$
\left\{\begin{array}{l}
B \boldsymbol{v}_{i+1}=v K \boldsymbol{v}_{i+1}+K \boldsymbol{v}_{i} \\
B K \boldsymbol{v}_{i+1}=(v-2 \omega) \boldsymbol{v}_{i+1}+\boldsymbol{v}_{i}
\end{array}\right.
$$

These two last equalities imply that the subspace generated by $\left\{\boldsymbol{v}_{i}, K \boldsymbol{v}_{i}\right\}_{i=1}^{l}$ is an invariant space of $B$. This concludes the proof.

Notation 3.8 We introduce the following

$$
\Gamma_{p}(a, s):=\left[\begin{array}{ccccc}
a & s & \cdots & 0 & 0 \\
0 & a & \cdots & 0 & 0 \\
\vdots & \vdots & \ddots & \vdots & \vdots \\
0 & 0 & \cdots & a & s \\
0 & 0 & \cdots & 0 & a
\end{array}\right],
$$

where $p$ denotes by the order of this matrix.

If $v \in \sigma\left(A_{\Gamma}\right)$ we denote by $E_{v}$ the (real) generalized spectral space corresponding to the eigenvalue $v$. 
Directly from Proposition 3.7 and by using notation above, we get that the restriction of $B$ onto the subspace $\widetilde{E}_{v}:=E_{v} \oplus E_{v-2 \omega}$ can be represented by the following $2 l \times 2 l$ matrix that in block matrix form can be written as follows

$$
\left.B\right|_{\widetilde{E}_{v}}=\left[\begin{array}{cc}
0_{l} & \Gamma_{l}(v, 1) \\
\Gamma_{l}(v-2 \omega,-1) & 0_{l}
\end{array}\right] .
$$

\section{3 $A_{\Gamma}$-Invariant $M_{\Gamma}$-Orthogonal Decomposition}

Let us start to introduce the following symmetric matrix

$$
\widehat{A}_{\Gamma}:=M_{\Gamma} A_{\Gamma}=D^{2} H(\xi)+\omega M_{\Gamma} .
$$

The rest of this section a bit technical in its own and the basic idea behind is to establish the behavior of the restriction of $\widehat{A}$ onto some subspaces constructed through the spectral subspaces (maybe generalized spectral subspaces) of $A_{\Gamma}$ which, as consequence of Corollary 3.10, are $\widehat{A}_{\Gamma^{-}}$orthogonal.

The next result provide a sufficient condition in order the generalized spectral subspaces relative to different and not conjugated eigenvalues to be $M_{\Gamma}$-orthogonal.

Lemma 3.9 Suppose that $v_{1}, v_{2} \in \sigma\left(A_{\Gamma}\right)$. If $v_{1} \neq \overline{v_{2}}$, then for every $v_{1} \in E_{v_{1}}$ and $\boldsymbol{v}_{2} \in E_{v_{2}}$ we have

$$
\left\langle\boldsymbol{v}_{1}, \boldsymbol{v}_{2}\right\rangle_{M_{\Gamma}}=\left\langle M_{\Gamma} \boldsymbol{v}_{1}, \boldsymbol{v}_{2}\right\rangle=0
$$

Proof We split the proof into two steps.

First step. We assume that $\boldsymbol{v}_{1}, \boldsymbol{v}_{2}$ are eigenvectors relative to the eigenvalues $v_{1}$ and $v_{2}$ respectively; thus, we have $A_{\Gamma} \boldsymbol{v}_{i}=v_{i} \boldsymbol{v}_{i}, i=1,2$. So, we have

$$
\begin{aligned}
& \left\langle\widehat{A}_{\Gamma} \boldsymbol{v}_{1}, \boldsymbol{v}_{2}\right\rangle=\left\langle M_{\Gamma} A_{\Gamma} \boldsymbol{v}_{1}, \boldsymbol{v}_{2}\right\rangle=\left\langle M_{\Gamma} v_{1} \boldsymbol{v}_{1}, \boldsymbol{v}_{2}\right\rangle=v_{1}\left\langle M_{\Gamma} \boldsymbol{v}_{1}, \boldsymbol{v}_{2}\right\rangle \text { and } \\
& \left\langle\widehat{A}_{1}, \boldsymbol{v}_{2}\right\rangle=\left\langle\boldsymbol{v}_{1}, M_{\Gamma} A_{\Gamma} \boldsymbol{v}_{2}\right\rangle=\left\langle\boldsymbol{v}_{1}, v_{2} M_{\Gamma} \boldsymbol{v}_{2}\right\rangle=\bar{v}_{2}\left\langle\boldsymbol{v}_{1}, M_{\Gamma} \boldsymbol{v}_{2}\right\rangle
\end{aligned}
$$

since $v_{1} \neq \overline{\nu_{2}}$, we get desired result.

Second step. We assume that $\boldsymbol{v}_{1}, \boldsymbol{v}_{2}$ are generalized eigenvectors and we consider the Jordan chains

$$
\begin{array}{ll}
A_{\Gamma} \boldsymbol{v}_{1}^{i+1}=v_{1} \boldsymbol{v}_{1}^{i+1}+\boldsymbol{v}_{1}^{i}, & \forall i \in\{0,1, \ldots, p\} \\
A_{\Gamma} \boldsymbol{v}_{2}^{j+1}=v_{2} \boldsymbol{v}_{2}^{j+1}+\boldsymbol{v}_{2}^{j}, & \forall j \in\{0,1, \ldots, q\}
\end{array}
$$

where $\boldsymbol{v}_{1}^{0}=\boldsymbol{v}_{2}^{0}=0$. By arguing as above, we get that

$$
\begin{aligned}
\left\langle M_{\Gamma} A_{\Gamma} \boldsymbol{v}_{1}^{i+1}, \boldsymbol{v}_{2}^{j+1}\right\rangle & =\left\langle M_{\Gamma} v_{1} \boldsymbol{v}_{1}^{i+1}+M_{\Gamma} \boldsymbol{v}_{1}^{i}, \boldsymbol{v}_{2}^{j+1}\right\rangle \\
& =v_{1}\left\langle M_{\Gamma} \boldsymbol{v}_{1}^{i+1}, \boldsymbol{v}_{2}^{j+1}\right\rangle+\left\langle M_{\Gamma} \boldsymbol{v}_{1}^{i}, \boldsymbol{v}_{2}^{j+1}\right\rangle . \text { Moreover }
\end{aligned}
$$




$$
\left\langle\boldsymbol{v}_{1}^{i+1}, M_{\Gamma} A_{\Gamma} \boldsymbol{v}_{2}^{j+1}\right\rangle=\bar{\nu}_{2}\left\langle M_{\Gamma} \boldsymbol{v}_{1}^{i+1}, \boldsymbol{v}_{2}^{j+1}\right\rangle+\left\langle M_{\Gamma} \boldsymbol{v}_{1}^{i}, \boldsymbol{v}_{2}^{j+1}\right\rangle
$$

So, taking the difference between the two equalities in Eq. (3.2), we get

$$
\begin{aligned}
0 & =\left\langle M_{\Gamma} A_{\Gamma} \boldsymbol{v}_{1}^{i+1}, \boldsymbol{v}_{2}^{j+1}\right\rangle-\left\langle\boldsymbol{v}_{1}^{i+1}, M_{\Gamma} A_{\Gamma} \boldsymbol{v}_{2}^{j+1}\right\rangle \\
& =\left(v_{1}-\bar{v}_{2}\right)\left\langle M_{\Gamma} \boldsymbol{v}_{1}^{i+1}, \boldsymbol{v}_{2}^{j+1}\right\rangle+\left\langle M_{\Gamma} \boldsymbol{v}_{1}^{i}, \boldsymbol{v}_{2}^{j+1}\right\rangle-\left\langle M_{\Gamma} \boldsymbol{v}_{1}^{i}, \boldsymbol{v}_{2}^{j+1}\right\rangle \\
& =\left(v_{1}-\bar{v}_{2}\right)\left\langle M_{\Gamma} \boldsymbol{v}_{1}^{i+1}, \boldsymbol{v}_{2}^{j+1}\right\rangle
\end{aligned}
$$

where the first equality follows by the fact that $A_{\Gamma}$ is $M_{\Gamma}$-symmetric and $M_{\Gamma}{ }^{\top}=M_{\Gamma}$. Since $v_{1}-\bar{v}_{2} \neq 0$, it follows that

$$
\left\langle M_{\Gamma} \boldsymbol{v}_{1}^{i+1}, \boldsymbol{v}_{2}^{j+1}\right\rangle=0 \quad \forall i \in\{0,1, \ldots, p\} \text { and } \forall j \in\{0,1, \ldots, q\} .
$$

To conclude the proof we argue by induction. Let $i+j=k$. So Eq. (3.4) is trivially true for $k=0$. Now, we suppose Eq. (3.4) holds true for $i+j=k \leq l$ and we want to prove that it is true for $i+j=k=l+1$. Now, by taking into account Eq. (3.3) and being $v_{1} \neq \bar{v}_{2}$, it readily follows that Eq. (3.4) holds true. This concludes the proof.

In particular generalized eigenspaces relative to different and not conjugated eigenvalues are $\widehat{A}_{\Gamma}$ orthogonal.

Corollary 3.10 Suppose that $v_{1}, v_{2} \in \sigma\left(A_{\Gamma}\right)$. If $v_{1} \neq \overline{v_{2}}$, then we have $\left\langle\widehat{A}_{\Gamma} \boldsymbol{v}_{1}, \boldsymbol{v}_{2}\right\rangle=0$ for all $\boldsymbol{v}_{1} \in E_{v_{1}}$ and $\boldsymbol{v}_{2} \in E_{v_{2}}$.

For $v \in \sigma\left(A_{\Gamma}\right)$, we set

$$
I_{v}:= \begin{cases}E_{v} \oplus E_{\bar{v}} & \text { if } v \notin \mathbb{R} \\ E_{v} & \text { otherwise. }\end{cases}
$$

Notation 3.11 Given any subspace $X \subset \mathbb{C}^{2 n}$, we denote by $\mathrm{n}_{-}\left(\left.\widehat{A}_{\Gamma}\right|_{X}\right)$ (resp. $\mathrm{n}_{+}\left(\left.\widehat{A}_{\Gamma}\right|_{X}\right)$ ), the dimension of the maximal negative (resp. positive) spectral subspace of the restriction of the quadratic form $\left\langle\widehat{A}_{\Gamma^{\cdot}}, \cdot\right\rangle$ onto $X$.

By the previous discussion, we can decompose the $\mathbb{C}^{2 n}=\mathbb{R}^{2 n} \otimes_{\mathbb{C}} \mathbb{C}$ into $A_{\Gamma^{-}}$ invariant , $M_{\Gamma}$-orthogonal subspaces; thus we have $\mathbb{C}^{2 n}=I_{\nu_{1}} \oplus \cdots \oplus I_{\nu_{l}}$, where $v_{i}$ are all distinct eigenvalues of $A_{\Gamma}$ with $\Im v_{i} \geq 0$.

Lemma 3.12 Let $v \in \sigma\left(A_{\Gamma}\right)$ and assume $v \neq 0$. Then the restriction

$$
\left.\left\langle\widehat{A}_{\Gamma} \cdot, \cdot\right\rangle\right|_{I_{v}}
$$

is non-degenerate. 
Proof We start to observe that ker $\widehat{A}_{\Gamma} \subseteq E_{0}=\operatorname{ker} A_{\Gamma} \cdot{ }^{2}$ Now, arguing by contradiction, we assume that $\left.\left\langle\widehat{A}_{\Gamma} \cdot, \cdot\right\rangle\right|_{I_{v}}$ is degenerate for some $v \neq 0$. Thus there exists $\boldsymbol{u} \in I_{v}$ and $\boldsymbol{u} 0 \neq$, such that

$$
\left\langle\widehat{A}_{\Gamma} \boldsymbol{u}, \boldsymbol{v}\right\rangle=0 \quad \text { for all } \boldsymbol{v} \in I_{\nu} \text {. }
$$

Since $\mathbb{C}^{2 n}$ is the direct sum of all different $I_{v^{\prime}}$, where $v^{\prime} \in \sigma\left(A_{\Gamma}\right)$, this implies (by invoking by Lemma 3.9) that $\left\langle\widehat{A}_{\Gamma} \boldsymbol{u}, \boldsymbol{v}\right\rangle=0$ for all $\boldsymbol{v} \in \mathbb{C}^{2 n}$. So $\boldsymbol{u} \in \operatorname{ker} \widehat{A}_{\Gamma}$ and in hence $\boldsymbol{u} \in \operatorname{ker} A_{\Gamma}=E_{0}$. Thus $v=0$ which is a contradiction. This concludes the proof.

The next result shed some light on the relation between the dimension of $I_{v}$ and the Morse index .

Lemma 3.13 Let $v \in \sigma\left(A_{\Gamma}\right)$, $\Im v>0$ and let $m_{v} \in \mathbb{N}$ be its algebraic multiplicity. Then

$$
\mathrm{n}_{-}\left[\left.\widehat{A}_{\Gamma}\right|_{I_{v}}\right]=m_{\nu}
$$

Proof By using Lemma 3.9, the quadratic form $\left\langle\widehat{A}_{\Gamma} \cdot, \cdot\right\rangle$ on $I_{v}=E_{v} \oplus E_{\bar{v}}$ can be represented in the block matrix form by $\left[\begin{array}{cc}0 & Y \\ Y^{\top} & 0\end{array}\right]$ for some $Y \in \operatorname{Mat}(v ; \mathbb{C})$. Moreover, by Lemma 3.12 we infer that $Y$ is non-degenerate and by this fact the conclusion readily follows.

By taking into account Corollary 3.10 as well as the additivity property of the inertia indices of $\widehat{A}$ with respect to the direct sum decomposition of the space into $\widehat{A}_{\Gamma}$-orthogonal subspaces, it follows that

$$
\mathrm{n}_{-}\left(\widehat{A}_{\Gamma}\right)=\sum_{i=1}^{l} \mathrm{n}_{-}\left(\widehat{A}_{\Gamma}^{v_{i}}\right)
$$

where we set $\widehat{A}_{\Gamma}^{v_{i}}:=\left.\left[D^{2} H(\xi)+\omega M_{\Gamma}\right]\right|_{I_{\nu_{i}}}$. Let $\left\{\boldsymbol{v}_{i}\right\}_{i=0}^{l}$ be a Jordan chain for for the generalized eigenspace $E_{v}$; thus

$$
\left\{\begin{array}{l}
A_{\Gamma} \boldsymbol{v}_{i+1}=v \boldsymbol{v}_{i+1}+\boldsymbol{v}_{i} \\
\boldsymbol{v}_{0}=0
\end{array}\right.
$$

By invoking Lemma 3.5, $\left\{K \boldsymbol{v}_{i}\right\}_{i=0}^{l}$ is a Jordan chain for the generalized eigenspace $E_{2 \omega-v}$ relative to $2 \omega-v$. More explicitly, the restriction of $A_{\Gamma}$ into the subspace generated by $\left\{K \boldsymbol{v}_{i}\right\}_{i=0}^{l}$ is given by $\Gamma_{l}(2 \omega-v,-1)$.

The next two results, Lemmas 3.14 and 3.15 will be very useful later on for computing the inertia indices of $\widehat{A}_{\Gamma}^{v}$ in terms of that of $M_{\Gamma}^{v}$. In Lemma 3.14 we investigate such a relation by restricting on a single Jordan block. In Lemma 3.15 we assume that there exists two different Jordan blocks corresponding to the same eigenvalue.

\footnotetext{
2 Actually if $M_{\Gamma}$ is positive definite it can be proved that also the converse inclusion holds.
} 
Lemma 3.14 Let $0 \neq v \in \sigma\left(A_{\Gamma}\right) \cap \mathbb{R}$ and let $\left\{\boldsymbol{v}_{i}\right\}_{i=0}^{l}$ be a Jordan chain for for the generalized eigenspace $E_{v}$. Under the previous notation we get that

$\left\langle M_{\Gamma} \boldsymbol{v}_{i}, \boldsymbol{v}_{j+1}\right\rangle=\left\langle M_{\Gamma} \boldsymbol{v}_{i+1}, \boldsymbol{v}_{j}\right\rangle$ and $\left\langle M_{\Gamma} \boldsymbol{v}_{i}, \boldsymbol{v}_{j}\right\rangle=0$ for $1 \leq j \leq l-i$ and $i=1, \ldots, l-1$

Proof By direct computation we infer that

$$
\begin{aligned}
\left\langle\widehat{A}_{\Gamma} \boldsymbol{v}_{i+1}, \boldsymbol{v}_{j+1}\right\rangle & =\left\langle M_{\Gamma} A_{\Gamma} \boldsymbol{v}_{i+1}, \boldsymbol{v}_{j+1}\right\rangle=\left\langle M_{\Gamma}\left(v \boldsymbol{v}_{i+1}+\boldsymbol{v}_{i}\right), \boldsymbol{v}_{j+1}\right\rangle \\
& =v\left\langle M_{\Gamma} \boldsymbol{v}_{i+1}, \boldsymbol{v}_{j+1}\right\rangle+\left\langle M_{\Gamma} \boldsymbol{v}_{i}, \boldsymbol{v}_{j+1}\right\rangle . \text { Moreover } \\
\left\langle\widehat{A}_{\Gamma} \boldsymbol{v}_{i+1}, \boldsymbol{v}_{j+1}\right\rangle & =\left\langle\boldsymbol{v}_{i+1}, \widehat{A}_{\Gamma} \boldsymbol{v}_{j+1}\right\rangle=\left\langle\boldsymbol{v}_{i+1}, M_{\Gamma} A_{\Gamma} \boldsymbol{v}_{j+1}\right\rangle \\
& =v\left\langle M_{\Gamma} \boldsymbol{v}_{i+1}, \boldsymbol{v}_{j+1}\right\rangle+\left\langle M_{\Gamma} \boldsymbol{v}_{i+1}, \boldsymbol{v}_{j}\right\rangle .
\end{aligned}
$$

By taking the difference of the first and last members in the previous equations, we get $\left\langle M_{\Gamma} \boldsymbol{v}_{i}, \boldsymbol{v}_{j+1}\right\rangle=\left\langle M_{\Gamma} \boldsymbol{v}_{i+1}, \boldsymbol{v}_{j}\right\rangle$. Being $\boldsymbol{v}_{0}=0$, we infer also that $\left\langle M_{\Gamma} \boldsymbol{v}_{i}, \boldsymbol{v}_{j}\right\rangle=0$ for every $1 \leq j \leq l-i$ and $i=1, \cdots, l-1$. This concludes the proof.

Lemma 3.15 Let $0 \neq v \in \sigma\left(A_{\Gamma}\right) \cap \mathbb{R}$ and we assume that $\left\{\boldsymbol{v}_{i}\right\}_{i=0}^{p}$ and $\left\{\boldsymbol{w}_{j}\right\}_{j=0}^{q}$ are two Jordan chains for the generalized eigenspaces relative to the same eigenvalue $v$ and such that $v_{0}=w_{0}=0$; furthermore we assume that $p \leq q$. Then we have $\left\langle M_{\Gamma} \boldsymbol{v}_{i}, \boldsymbol{w}_{j+1}\right\rangle=\left\langle M_{\Gamma} \boldsymbol{v}_{i+1}, \boldsymbol{w}_{j}\right\rangle$ and $\left\langle M_{\Gamma} \boldsymbol{v}_{i}, \boldsymbol{w}_{j}\right\rangle=0$ for $1 \leq i+j \leq q$.

Proof By a direct computation we get $\left\langle M_{\Gamma} \boldsymbol{v}_{i}, \boldsymbol{w}_{j+1}\right\rangle=\left\langle M_{\Gamma} \boldsymbol{v}_{i+1}, \boldsymbol{w}_{j}\right\rangle$. Since $\boldsymbol{v}_{0}=$ $\boldsymbol{w}_{0}=0$ and being $p \leq q$, then we have $\left\langle M_{\Gamma} \boldsymbol{v}_{i}, \boldsymbol{w}_{j}\right\rangle=0$ for $1 \leq i+j \leq q$. This concludes the proof.

\section{Proof of Main Results}

This section is devoted to prove the main results of this paper. The first result provides a characterization of the spectral stability of a relative equilibrium $z$ in terms of a spectral condition on the central configuration $\xi$.

Proof of Theorem 1 The proof of this result result direct follows by the first claim in Lemma 3.2. In fact, $\mu$ is an eigenvalue of $M_{\Gamma}^{-1} D^{2} H(\xi)$ if and only if $\lambda=\sqrt{\mu^{2}-\omega^{2}}$ is an eigenvalue of $B$. By definition, $z$ is spectrally stable if and only if the spectrum of $B$ is purely imaginary or which is the same that $\mu^{2}-\omega^{2} \leq 0$. This concludes the proof.

The next result shed provides a cler relation intertwining the spectral condition on the central configuration generating the relative equilibrium seen as critical point of the Hamiltonian on the shape pseudo-sphere and the dynamical (stability) properties of it. Roberts in Theorem 3.3 of [7] characterizes linearly stable relative equilibria in terms of the minimality properties that the central configuration (originating such an equilibrium) possesses. 
Theorem 4.1 [7] We assume that for every $j, \Gamma_{j}>0$. Then a relative equilibrium $z$ is linearly stable if and only if it is a non-degenerate minimum of $H$ restricted to the shape-pseudo-sphere.

Thus, by Theorem 4.1, the linear stability of a relative equilibrium is equivalent to the fact that the central configuration generating it has a vanishing Morse index and it is non-degenerate (meaning that the kernel dimension of the Hessian of the Hamiltonian restricted to the shape pseudo-sphere vanishes identically). However this result is valid only under the assumption that all circulations have the same sign. In Theorem 2, by using the analysis performed in the previous sections, we are able to remove the condition on the circulations' sign admitting any kind of (non-vanishing) circulation and we provide a relation between the spectral stability of a relative equilibrium and the Morse index of the central configuration generating it. As Corollary of this result, we complement the aforementioned Theorem 4.1.

Before giving the proof of this result, we observe that if all circulations strengths have all the same sign (for instance, positive), then $M_{\Gamma}$ is positive definite (thus $\mathrm{n}_{-}\left(M_{\Gamma}\right)=0$ ) and by Eq. (2.9) in particular $\omega$ is positive. Thus by the first claim of Theorem 2, we conclude that $\xi$ is a minimum (maybe degenerate).

Corollary 4.2 If $\Gamma_{j}>0$ for all $j$, and we assume that $z$ is a spectral stable non-degenerate relative equilibrium. Then the central configuration $\xi$ is a (maybe degenerate) minimum of $H$.

Proof The proof of the first claim follows by the above discussion.

Before providing the proof of Theorem 2, we start proving the following technical result.

Lemma 4.3 Let $v$ be a non-zero real eigenvalue of matrix $\widehat{A}_{\Gamma}$, then we have that

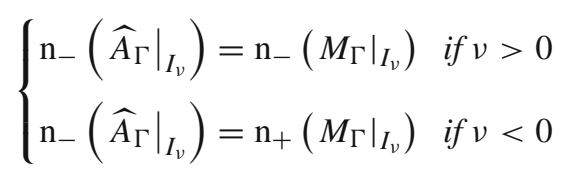

Proof We assume that there exist two different Jordan blocks $\Gamma_{1}(v), \Gamma_{2}(v)$ corresponding to same eigenvalue $v$ and, as before, we denote by $\left\{\boldsymbol{v}_{i}\right\}_{i=0}^{p}$ and $\left\{\boldsymbol{w}_{j}\right\}_{j=0}^{q}$ the Jordan chains corresponding to these Jordan blocks. Let us consider the following matrix block decomposition

$$
\widehat{A}(v)=\left[\begin{array}{ll}
A_{1} & A_{2} \\
A_{2}^{T} & A_{3}
\end{array}\right] \quad \text { and } \quad M_{\Gamma}(v)=\left[\begin{array}{ll}
M_{1} & M_{2} \\
M_{2}^{T} & M_{3}
\end{array}\right],
$$

where $\left(A_{1}\right)_{i j}=\left\langle\widehat{A} \boldsymbol{v}_{i}, \boldsymbol{v}_{j}\right\rangle,\left(A_{2}\right)_{i j}=\left\langle\widehat{A} \boldsymbol{v}_{i}, \boldsymbol{w}_{j}\right\rangle,\left(A_{3}\right)_{i j}=\left\langle\widehat{A} \boldsymbol{w}_{i}, \boldsymbol{w}_{j}\right\rangle, \quad\left(M_{1}\right)_{i j}=$ $\left\langle M \boldsymbol{v}_{i}, \boldsymbol{v}_{j}\right\rangle,\left(M_{2}\right)_{i j}=\left\langle\boldsymbol{M} \boldsymbol{v}_{i}, \boldsymbol{w}_{j}\right\rangle$ and finally $\left(M_{3}\right)_{i j}=\left\langle M \boldsymbol{w}_{i}, \boldsymbol{w}_{j}\right\rangle$. By Lemma 3.14, one immediately get that the $p \times p$ block $A_{1}$ is given by:

$$
A_{1}=\left\langle\widehat{A} \boldsymbol{v}_{i+1}, \boldsymbol{v}_{j+1}\right\rangle=v\left\langle M_{\Gamma} \boldsymbol{v}_{i+1}, \boldsymbol{v}_{j+1}\right\rangle+\left\langle M_{\Gamma} \boldsymbol{v}_{i}, \boldsymbol{v}_{j+1}\right\rangle
$$




$$
=\left[\begin{array}{ccccc}
0 & 0 & \cdots & 0 & v\left\langle M_{\Gamma} \boldsymbol{v}_{1}, \boldsymbol{v}_{p}\right\rangle \\
0 & 0 & \cdots & v\left\langle M_{\Gamma} \boldsymbol{v}_{1}, \boldsymbol{v}_{p}\right\rangle & \left\langle M_{\Gamma} \boldsymbol{v}_{1}+v M_{\Gamma} \boldsymbol{v}_{2}, \boldsymbol{v}_{p}\right\rangle \\
\vdots & \vdots & \ddots & \vdots & \vdots \\
0 & v\left\langle M_{\Gamma} \boldsymbol{v}_{1}, \boldsymbol{v}_{p}\right\rangle & \cdots & \left\langle M_{\Gamma} \boldsymbol{v}_{p-3}+v M_{\Gamma} \boldsymbol{v}_{p-2}, \boldsymbol{v}_{p}\right\rangle & \left\langle M_{\Gamma} \boldsymbol{v}_{p-2}+v M_{\Gamma} \boldsymbol{v}_{p-1}, \boldsymbol{v}_{p}\right\rangle \\
v\left\langle M_{\Gamma} \boldsymbol{v}_{1}, \boldsymbol{v}_{p}\right\rangle\left\langle M_{\Gamma} \boldsymbol{v}_{1}+v M_{\Gamma} \boldsymbol{v}_{2}, \boldsymbol{v}_{p}\right\rangle & \cdots & \left\langle M_{\Gamma} \boldsymbol{v}_{p-2}+v M_{\Gamma} \boldsymbol{v}_{p-1}, \boldsymbol{v}_{p}\right\rangle & \left\langle M_{\Gamma} \boldsymbol{v}_{p-1}+v M_{\Gamma} \boldsymbol{v}_{p}, \boldsymbol{v}_{p}\right\rangle
\end{array}\right]
$$

and

$$
M_{1}=\left[\begin{array}{ccccc}
0 & 0 & \cdots & 0 & \left\langle M_{\Gamma} \boldsymbol{v}_{1}, \boldsymbol{v}_{p}\right\rangle \\
0 & 0 & \cdots & \left\langle M_{\Gamma} \boldsymbol{v}_{1}, \boldsymbol{v}_{p}\right\rangle & \left\langle M_{\Gamma} v_{2}, \boldsymbol{v}_{p}\right\rangle \\
\vdots & \vdots & \ddots & \vdots & \vdots \\
0 & \left\langle M_{\Gamma} \boldsymbol{v}_{1}, \boldsymbol{v}_{p}\right\rangle & \cdots & \left.M_{\Gamma} \boldsymbol{v}_{p-2}, \boldsymbol{v}_{p}\right\rangle & \left\langle M_{\Gamma} \boldsymbol{v}_{p-1}, \boldsymbol{v}_{p}\right\rangle \\
\left\langle M_{\Gamma} \boldsymbol{v}_{1}, \boldsymbol{v}_{p}\right\rangle & \left.M_{\Gamma} \boldsymbol{v}_{2}, \boldsymbol{v}_{p}\right\rangle & \cdots & \left.M_{\Gamma} \boldsymbol{v}_{p-1}, \boldsymbol{v}_{p}\right\rangle & \left\langle M_{\Gamma} \boldsymbol{v}_{p}, \boldsymbol{v}_{p}\right\rangle
\end{array}\right] .
$$

It is readily seen that the matrix $A_{1}$ given in Eq. (4.1) can be written in equivalent form, as follows

$$
A_{1}=M_{1} \Gamma_{p}(\nu, 1)
$$

Analogously, we have that

$$
A_{3}=M_{3} \Gamma_{q}(v, 1) .
$$

By Lemma 3.15, one gets that the $p \times q$ block $A_{2}$ is given by

$$
\begin{aligned}
A_{2} & =\left\langle\widehat{A} \boldsymbol{v}_{i+1}, \boldsymbol{w}_{j+1}\right\rangle=v\left\langle M_{\Gamma} \boldsymbol{v}_{i+1}, \boldsymbol{w}_{j+1}\right\rangle+\left\langle M_{\Gamma} \boldsymbol{v}_{i}, \boldsymbol{w}_{j+1}\right\rangle \\
& =\left[\begin{array}{ccccc}
0 & 0 & \cdots & 0 & v\left\langle M_{\Gamma} \boldsymbol{v}_{1}, \boldsymbol{v}_{q}\right\rangle \\
0 & 0 & \cdots & v\left\langle M_{\Gamma} \boldsymbol{v}_{1}, \boldsymbol{v}_{q}\right\rangle & \left\langle M_{\Gamma} \boldsymbol{v}_{1}+v M_{\Gamma} \boldsymbol{v}_{2}, \boldsymbol{v}_{q}\right\rangle \\
\vdots & \vdots & \ddots & \vdots & \vdots \\
0 & v\left\langle M_{\Gamma} \boldsymbol{v}_{1}, \boldsymbol{v}_{q}\right\rangle & \cdots & \left\langle M_{\Gamma} \boldsymbol{v}_{p-3}+v M_{\Gamma} \boldsymbol{v}_{p-2}, \boldsymbol{v}_{q}\right\rangle & \left\langle M_{\Gamma} \boldsymbol{v}_{p-2}+v M_{\Gamma} \boldsymbol{v}_{p-1}, \boldsymbol{v}_{q}\right\rangle \\
v\left\langle M_{\Gamma} \boldsymbol{v}_{1}, \boldsymbol{v}_{q}\right\rangle\left\langle M_{\Gamma} \boldsymbol{v}_{1}+v M_{\Gamma} \boldsymbol{v}_{2}, \boldsymbol{v}_{q}\right\rangle & \cdots & \left\langle M_{\Gamma} \boldsymbol{v}_{p-2}+v M_{\Gamma} \boldsymbol{v}_{p-1}, \boldsymbol{v}_{q}\right\rangle & \left\langle M_{\Gamma} \boldsymbol{v}_{p-1}+v M_{\Gamma} \boldsymbol{v}_{p}, \boldsymbol{v}_{q}\right\rangle
\end{array}\right]
\end{aligned}
$$

and

$$
M_{2}=\left[\begin{array}{ccccc}
0 & 0 & \cdots & 0 & \left\langle M_{\Gamma} \boldsymbol{v}_{1}, \boldsymbol{v}_{q}\right\rangle \\
0 & 0 & \cdots & \left\langle M_{\Gamma} \boldsymbol{v}_{1}, \boldsymbol{v}_{q}\right\rangle & \left\langle M_{\Gamma} \boldsymbol{v}_{2}, \boldsymbol{v}_{q}\right\rangle \\
\vdots & \vdots & \ddots & \vdots & \vdots \\
0 & \left\langle M_{\Gamma} \boldsymbol{v}_{1}, \boldsymbol{v}_{q}\right\rangle & \cdots & \left.M_{\Gamma} \boldsymbol{v}_{p-2}, \boldsymbol{v}_{q}\right\rangle & \left\langle M_{\Gamma} \boldsymbol{v}_{p-1}, \boldsymbol{v}_{q}\right\rangle \\
\left.M_{\Gamma} \boldsymbol{v}_{2}, \boldsymbol{v}_{q}\right\rangle & \cdots & \left.M_{\Gamma} \boldsymbol{v}_{p-1}, \boldsymbol{v}_{q}\right\rangle & \left\langle M_{\Gamma} \boldsymbol{v}_{p}, \boldsymbol{v}_{q}\right\rangle
\end{array}\right]
$$

so, as before, by Eqs. (4.4) and (4.5) imply that

$$
A_{2}=M_{2} \Gamma_{q}(v, 1) \text {. }
$$

Similarly for the term

$$
A_{2}^{\top}=M_{2}^{T} \Gamma_{p}(v, 1) .
$$

Thus (4.2), (4.3), (4.6) and finally (4.7) imply that

$$
\widehat{A}_{\Gamma}^{v}=M_{\Gamma}^{v} \operatorname{diag}\left[\Gamma_{p}(v, 1), \Gamma_{q}(v, 1)\right] .
$$


Case 1. If $v>0$, we define the (analytic) path of symmetric matrices pointwise given by

$$
f(t):=M_{\Gamma}^{\nu}\left(\begin{array}{cc}
\Gamma_{p}(t, 1) & 0 \\
0 & \Gamma_{q}(t, 1)
\end{array}\right)
$$

parametrized by the interval $[1, v]$, if $v>1$ and by $[v, 1]$, if $v<1$. Moreover, we let

$$
g(s):=M_{\Gamma}^{v}\left(\begin{array}{cc}
\Gamma_{p}(1, s) & 0 \\
0 & \Gamma_{q}(1, s)
\end{array}\right) \quad \text { for } s \in[0,1] .
$$

If an eigenvalue of $f(t)$ (resp. $g(s)$ ) changes sign, than det $f(t)=0$ (resp. $\operatorname{det}(g(s)=0)$. However, it is immediate to see that this cannot occur. We observe that the composition of the two paths $f$ and $g$ is a continuous path joining the matrices $M_{\Gamma}^{v}$ matrix to $\widehat{A}_{\Gamma}^{v}$. By this argument it then follows that both matrices belong to the same connected component and in particular the inertia indices coincide; thus in symbols, we have

$$
\begin{aligned}
& \mathrm{n}_{-}(\widehat{A}(v))=\mathrm{n}_{-}\left(M_{\Gamma}(v)\right), \\
& \mathrm{n}_{+}(\widehat{A}(v))=\mathrm{n}_{+}\left(M_{\Gamma}(v)\right) .
\end{aligned}
$$

Case 2. If $v<0$, as before, we define the path of symmetric matrices

$$
f(t):=M_{\Gamma}^{v}\left(\begin{array}{cc}
\Gamma_{p}(t, 1) & 0 \\
0 & \Gamma_{q}(t, 1)
\end{array}\right)
$$

parametrized by the interval $[v,-1]$ if $v<-1$ and by $[-1, v]$ if $v>-1$. As before, we let

$$
g(s):=M_{\Gamma}^{v}\left(\begin{array}{cc}
\Gamma_{p}(-1,-s) & 0 \\
0 & \Gamma_{q}(-1,-s)
\end{array}\right)
$$

where $s \in[-1,0]$. Arguing as before, we get

$$
\begin{aligned}
& \mathrm{n}_{-}\left(\widehat{A}_{\Gamma}^{v}\right)=\mathrm{n}_{+}\left(M_{\Gamma}^{v}\right), \\
& \mathrm{n}_{+}\left(\widehat{A}_{\Gamma}^{v}\right)=\mathrm{n}_{-}\left(M_{\Gamma}^{v}\right) .
\end{aligned}
$$

Proof of Theorem 2 Since $z$ is non-degenerate and spectrally stable relative equilibrium, then by invoking Lemma 3.2, we get that

$$
\sigma\left(A_{\Gamma}\right) \subset \mathbb{R} \bigcup\{\omega+i x \mid x \in \mathbb{R}\}
$$

We notice that

$$
\widehat{A}_{\Gamma}(\xi)=D^{2} H(\xi) \xi+\omega M_{\Gamma} \xi=M_{\Gamma}\left(M_{\Gamma}^{-1} D^{2} H(\xi)-\omega I+2 \omega I\right) \xi=2 \omega M_{\Gamma} \xi
$$


and from property (iii) of Lemma 2.6 we have

$$
\begin{aligned}
\widehat{A}_{\Gamma}(K \xi)=D^{2} H(z) K \xi+\omega M_{\Gamma} K \xi & =M_{\Gamma}\left(M_{\Gamma}^{-1} D^{2} H(z)+\omega I\right) K \xi \\
& =M_{\Gamma} K\left(-M_{\Gamma}^{-1} D^{2} H(z)+\omega I\right) \xi=0
\end{aligned}
$$

If $W=\operatorname{span}(\xi, K \xi)$, then we get that

$$
\begin{aligned}
\left.\widehat{A}_{\Gamma}\right|_{W} & =\left[\begin{array}{cc}
\left\langle\widehat{A}_{\Gamma}(\boldsymbol{\xi}), \boldsymbol{\xi}\right\rangle & \left\langle\widehat{A}_{\Gamma}(\boldsymbol{\xi}), K \boldsymbol{\xi}\right\rangle \\
\left\langle\widehat{A}_{\Gamma}(K \boldsymbol{\xi}), \boldsymbol{\xi}\right\rangle & \left\langle\widehat{A}_{\Gamma}(K \boldsymbol{\xi}), K \boldsymbol{\xi}\right\rangle
\end{array}\right] \\
& =\left[\begin{array}{cc}
\left\langle\omega M_{\Gamma} \boldsymbol{\xi}, \boldsymbol{\xi}\right\rangle\left\langle\boldsymbol{\xi}, \widehat{A}_{\Gamma}(K \boldsymbol{\xi})\right\rangle \\
0 & 0
\end{array}\right] \\
& =\left[\begin{array}{cc}
2 \omega\left\langle M_{\Gamma} \xi, \xi\right\rangle & 0 \\
0 & 0
\end{array}\right] .
\end{aligned}
$$

Thus

$$
\mathrm{n}_{-}\left(\left.\widehat{A}(\xi)\right|_{W}\right)= \begin{cases}1 & \text { if } 2 \omega\left\langle M_{\Gamma} \xi, \xi\right\rangle<0 \\ 0 & \text { if } 2 \omega\left\langle M_{\Gamma} \xi, \xi\right\rangle>0\end{cases}
$$

In conclusion, we get

$$
\mathrm{n}_{-}\left(\left.\widehat{A}(\boldsymbol{\xi})\right|_{W}\right)=\operatorname{sign}\left(2 \omega\left\langle M_{\Gamma} \boldsymbol{\xi}, \boldsymbol{\xi}\right\rangle\right)
$$

where sign stands for the sign. Let us now consider the $\widehat{A}_{\Gamma}$-orthogonal $M_{\Gamma}$-orthogonal invariant decomposition of the full (complexified) phase space $\mathbb{C}^{2 n}$. Since $\mathbb{C}^{2 n}=$ $W \oplus W^{\perp}=I_{0} \oplus I_{2 \omega} \oplus_{i}\left(I_{\nu_{i}} \oplus_{i=1}^{l} I_{\nu_{2 \omega-v_{i}}}\right)$, where $v_{i} \in \sigma\left(A_{\Gamma}\right) \backslash\{0,2 \omega\}$. So we have that

$$
\mathrm{n}_{-}\left(\left.\widehat{A}(\xi)\right|_{W^{\perp}}\right)=\sum_{i=1}^{l}\left(\mathrm{n}_{-}\left(\left.\widehat{A}(\xi)\right|_{I_{v_{i}}}\right)+\mathrm{n}_{-}\left(\left.\widehat{A}(\xi)\right|_{I_{2 \omega-v_{i}}}\right)\right)
$$

and

$$
\mathrm{n}_{-}\left(\left.M_{\Gamma}\right|_{W^{\perp}}\right)=\sum_{i=1}^{l}\left(\mathrm{n}_{-}\left(\left.M_{\Gamma}\right|_{I_{v_{i}}}\right)+\mathrm{n}_{-}\left(\left.M_{\Gamma}\right|_{I_{2 \omega-v_{i}}}\right)\right) .
$$

We let $v \in \sigma\left(A_{\Gamma}\right) \backslash\{0,2 \omega\}$ and we consider separately the two cases: $v \in \mathbb{R}$ and $\nu \in \mathbb{C} \backslash \mathbb{R}$.

If $v \in \mathbb{R}$ as consequence of the first item in Lemma 3.2 only two cases can occur:

- Subcase 1: $\omega$ positive and $0<v<2 \omega$. (In particular $2 \omega-v>0$ and $v>0$ );

- Subcase 2: $v \in \mathbb{R}, \omega$ negative and $2 \omega<v<0$. (In particular $2 \omega-v<0$ and $v<0)$.

By Lemma 4.3, we have that

$$
\begin{cases}\mathrm{n}_{-}\left(\left.\widehat{A}_{\Gamma}\right|_{I_{\nu}}\right)=\mathrm{n}_{-}\left(\left.M_{\Gamma}\right|_{I_{\nu}}\right) & \\ \mathrm{n}_{-}\left(\left.\widehat{A}_{\Gamma}\right|_{I_{\nu}}\right)=\mathrm{n}_{+}\left(\left.M_{\Gamma}\right|_{I_{\nu}}\right) & \text { [Subcase 1] }\end{cases}
$$


Case 2: $v \in\{i x+\omega \mid x \in \mathbb{R}\} \subset \mathbb{C} \backslash \mathbb{R}$. By using Lemma 3.13, we already know that $\mathrm{n}_{-}\left(\widehat{A}_{\Gamma}^{v}\right)=m_{v}$, where $m_{v}$ is the algebraic multiplicities of the eigenvalue $v$. Moreover, from Corollary 3.10, the matrix $M_{\Gamma}^{\nu}$ can be represented as follows

$$
\left[\begin{array}{cc}
0 & Y \\
Y^{\top} & 0
\end{array}\right]
$$

for some $Y \in \mathscr{L}\left(E_{\nu}, E_{\bar{v}}\right)$.

Now, since $M_{\Gamma}^{v}$ is non-degenerate, it readily follows that $\mathrm{n}_{-}\left(M_{\Gamma}^{v}\right)=\mathrm{n}_{+}\left(M_{\Gamma}^{v}\right)=$ $m_{\nu}$. In conclusion, we get $\mathrm{n}_{-}\left(\widehat{A}^{v}\right)=\mathrm{n}_{-}\left(M_{\Gamma}^{v}\right)=\mathrm{n}_{+}\left(M^{v}\right)=m_{\nu}$. This concludes the proof.

We let $N_{C}$ be the total number of eigenvalues of the matrix $A_{\Gamma}$ having positive imaginary part and counted according to their algebraic multiplicities. By using item 2. in Lemma 3.2 we are entitled to define $N_{0}$ as the total number (counted according to their algebraic multiplicities) of real eigenvalues $\tilde{\mu}$ of the matrix $A_{\Gamma}$ such that

$$
\tilde{\mu} \in \begin{cases}(2 \omega,+\infty) & \text { if } \omega>0 \\ (-\infty, 2 \omega) & \text { if } \omega<0\end{cases}
$$

Being due to the fact $\lambda \in \sigma(B)$ if and only if $\nu_{ \pm}:= \pm \sqrt{\lambda^{2}+\omega^{2}}+\omega \in \sigma\left(A_{\Gamma}\right), N_{0}$ is also equal to the total number (counted according to their algebraic multiplicities )of the positive real eigenvalues of the stability matrix $B$.

Theorem 4.4 Let $z$ be a relative equilibrium. Then we have

$$
\mathrm{n}_{-}\left(\widehat{A_{\Gamma}}\right)= \begin{cases}\sum_{v \in[0,2 \omega]} \mathrm{n}_{-}\left(\left.M_{\Gamma}\right|_{I_{v}}\right)+N_{C}+N_{0}, & \text { if } \omega>0, \\ \sum_{v \in[2 \omega, 0]} \mathrm{n}_{+}\left(\left.M_{\Gamma}\right|_{I_{v}}\right)+N_{C}+N_{0}, & \text { if } \omega<0 .\end{cases}
$$

Proof We only prove the case of $\omega>0$ being the other completely analogous. As already observed in Eq. (3.5) we get that

$$
\mathrm{n}_{-}\left(\widehat{A}_{\Gamma}\right)=\sum_{i=1}^{l} \mathrm{n}_{-}\left(\widehat{A}_{\Gamma}^{v_{i}}\right)
$$

By using Lemma 3.13, we get

$$
\sum_{\Im v>0} \mathrm{n}_{-}\left(\widehat{A}_{\Gamma}^{v_{i}}\right)=N_{C}
$$

Let $v>2 \omega$ be a (positive real) eigenvalue of the matrix $A_{\Gamma}$; then $2 \omega-v$ is as well an eigenvalue of the modified Hessian $A_{\Gamma}$ having the same algebraic multiplicity. By Lemma 3.5 and taking advantage that $K^{2}=I$ as well as $K M_{\Gamma}=-M_{\Gamma} K$ by a straightforward calculation, we get that

$$
\mathrm{n}_{ \pm}\left(\left.M_{\Gamma}\right|_{I_{\nu}}\right)=\mathrm{n}_{ \pm}\left(\left.M_{\Gamma}\right|_{I_{2 \omega-\nu}}\right) .
$$


By using Lemma 4.3, we infer also that

$$
\mathrm{n}_{-}\left(\left.\widehat{A_{\Gamma}}\right|_{I_{\nu}}\right)+\mathrm{n}_{-}\left(\left.\widehat{A_{\Gamma}}\right|_{I_{2 \omega-v}}\right)=\mathrm{n}_{-}\left(\left.M_{\Gamma}\right|_{I_{v}}\right)+n_{+}\left(\left.M_{\Gamma}\right|_{I_{2 \omega-v}}\right)=m_{\nu}
$$

where, as before, $m_{v}$ denotes by the algebraic multiplicity of the eigenvalue $v$. This implies that

$$
\sum_{\nu>2 \omega}\left[\mathrm{n}_{-}\left(\left.\widehat{A_{\Gamma}}\right|_{I_{\nu}}\right)+\mathrm{n}_{-}\left(\left.\widehat{A_{\Gamma}}\right|_{I_{2 \omega-\nu}}\right)\right]=N_{0}
$$

So by taking into account Eqs. (3.5)-(4.9) as well as Lemma 4.3, we get that

$$
\mathrm{n}_{-}\left(\widehat{A_{\Gamma}}\right)=\sum_{\nu \in[0,2 \omega]} \mathrm{n}_{-}\left(\left.M_{\Gamma}\right|_{I_{\nu}}\right)+N_{C}+N_{0}
$$

This concludes the proof.

Remark 4.5 In [8], author considered the case of positive circulations; thus in particular $M_{\Gamma}$ is positive definite. By using the Sylvester's inertia Theorem, it follows that $\mathrm{n}_{-}\left(\widehat{A}_{\Gamma}\right)=\mathrm{n}_{-}\left(A_{\Gamma}\right)$.

[8, Item (i) in Lemma 3.3] shows that the eigenvalues of $A_{\Gamma}$ come in pairs and are of the form $\left(v_{i}, 2 \omega-v_{i}\right)$. This property implies the existence of an upper bound for the Morse index of the relative equilibria. As already observed by Roberts in [8], this upper bound already appeared in [12].

Theorem 4.6 [8, Theorem 3.4] Under the above notation we assume that $\Gamma_{j}>0$ are positive for every $j$ and let $z$ be a relative equilibrium. Then

$$
\mathrm{n}_{-}\left(\widehat{A_{\Gamma}}\right) \leq n-2
$$

with equality holding whenever $z$ is a collinear configuration.

Theorem 4.7 [8, Theorem 3.5] Under the above notation we assume that $\Gamma_{i}>0$ for all $i$ and let $z$ be a relative equilibrium. Then the Morse index of $z$ is equal to the number of pairs of real (nonzero) eigenvalues $\pm \lambda$ of the corresponding periodic solution.

Remark 4.8 It is worth noticing that for positive circulations, then the matrix $M_{\Gamma}$ is positive definite and the matrix $A_{\Gamma}$ is diagonalizable on the reals; so $N_{C}=0$. Furthermore by Lemma 3.9 and being $M_{\Gamma}$ positive definite, we get also that $\mathrm{n}_{-}\left(\left.M_{\Gamma}\right|_{I_{v}}\right)=0$ for $v>0$. This, in particular, yields $\sum_{v \in[0,2 \omega]} \mathrm{n}_{-}\left(\left.M_{\Gamma}\right|_{I_{v}}\right)=0$. So Theorem 4.6 as well as Theorem 4.7 can be regarded as two directly corollaries of Theorem 4.4.

Theorem 4.7 yields a direct correspondence between the Morse index and the instability of the relative equilibrium. However, such a relation failed in the case of mixed-sign circulations. 


\section{Some Symmetric Examples}

This section is devoted to the application of Theorem 2 to some specific examples of relative equilibria in the planar $N$-vortex problem.

\subsection{The Equilateral Triangle}

We begin with the well-known equilateral triangle solution in the three-vortex problem. Placing three vortices of any strength at the vertices of an equilateral triangle yields a relative equilibrium. Synge [9], showed that the corresponding relative equilibrium is linearly stable if and only if $L>0$. (Cfr. [7, Sect. 4] for further details).

Theorem 5.1 Given any three circulations $\Gamma_{1}, \Gamma_{2} \Gamma_{3}$, let

$$
\widehat{\xi}_{1}=(1,0), \widehat{\xi}_{2}=\left(-\frac{1}{2}, \frac{\sqrt{3}}{2}\right), \widehat{\xi}_{3}=\left(-\frac{1}{2},-\frac{\sqrt{3}}{2}\right),
$$

and let $\widehat{\boldsymbol{c}}=\sum_{i=3}^{3} \Gamma_{i} \widehat{\boldsymbol{z}}_{i}$. We assume that $L>0$ and let $\boldsymbol{\xi}=\left(\boldsymbol{\xi}_{1}, \boldsymbol{\xi}_{2}, \boldsymbol{\xi}_{3}\right)$, for $\boldsymbol{\xi}_{i}=\widehat{\boldsymbol{\xi}}_{i}-\widehat{\boldsymbol{c}}$. Then

$$
\mathrm{n}_{-}\left(\widehat{A}_{\Gamma}(\xi)\right)=\left\{\begin{array}{ll}
0 & \text { if } \Gamma_{1}, \Gamma_{2}, \Gamma_{3} \text { have the same sign } \\
1 & \text { if there is only one } \Gamma_{i}<0 \\
2 & \text { otherwise }
\end{array} .\right.
$$

Proof As proved by author in [9], we get that $\boldsymbol{\xi}=\left(\boldsymbol{\xi}_{1}, \boldsymbol{\xi}_{2}, \boldsymbol{\xi}_{3}\right)$ is a linearly stable iff $L>0$; moreover, the angular velocity $\omega=\frac{\Gamma}{3}$. We note that the vortex positions $\boldsymbol{\xi}_{i}=\widehat{\boldsymbol{\xi}}_{i}-\widehat{\boldsymbol{c}}$ have center of vorticity at the origin.

By an explicit calculation, we get

$$
\begin{aligned}
\widehat{\boldsymbol{c}}=\frac{1}{\Gamma} \sum_{i=1}^{3} \Gamma_{i} \boldsymbol{\xi}_{i} & =\frac{1}{\Gamma}\left(\Gamma_{1}(1,0)+\Gamma_{2}\left(-\frac{1}{2}, \frac{\sqrt{3}}{2}\right)+\Gamma_{3}\left(-\frac{1}{2},-\frac{\sqrt{3}}{2}\right)\right) \\
& =\left(\frac{\Gamma_{1}}{\Gamma}-\frac{\Gamma_{1}+\Gamma_{2}}{2 \Gamma}, \frac{\sqrt{3}}{2 \Gamma}\left(\Gamma_{2}-\Gamma_{3}\right)\right) \\
\boldsymbol{\xi}_{1}=\widehat{\boldsymbol{\xi}}_{1}-\widehat{\boldsymbol{c}} & =(1,0)-\left(\frac{\Gamma_{1}}{\Gamma}-\frac{\Gamma_{3}+\Gamma_{2}}{2 \Gamma}, \frac{\sqrt{3}}{2 \Gamma}\left(\Gamma_{2}-\Gamma_{3}\right)\right) \\
& =\left(\frac{3\left(\Gamma_{3}+\Gamma_{2}\right)}{2 \Gamma},-\frac{\sqrt{3}}{2 \Gamma}\left(\Gamma_{2}-\Gamma_{3}\right)\right) \\
\boldsymbol{\xi}_{2}=\widehat{\boldsymbol{\xi}}_{2}-\widehat{\boldsymbol{c}} & =\left(-\frac{1}{2}, \frac{\sqrt{3}}{2}\right)-\left(\frac{\Gamma_{1}}{\Gamma}-\frac{\Gamma_{1}+\Gamma_{2}}{2 \Gamma}, \frac{\sqrt{3}}{2 \Gamma}\left(\Gamma_{2}-\Gamma_{3}\right)\right)
\end{aligned}
$$




$$
\begin{aligned}
& =\left(-\frac{1}{2}-\frac{\Gamma_{1}}{\Gamma}+\frac{\Gamma_{1}+\Gamma_{2}}{2 \Gamma}, \frac{\sqrt{3}}{2}-\frac{\sqrt{3}}{2 \Gamma}\left(\Gamma_{2}-\Gamma_{3}\right)\right) \\
& =\left(-\frac{3 \Gamma_{1}}{2 \Gamma}, \frac{\sqrt{3}}{2 \Gamma}\left(\Gamma_{1}+2 \Gamma_{3}\right)\right) \\
\boldsymbol{\xi}_{3}=\widehat{\boldsymbol{\xi}}_{3}-\widehat{\boldsymbol{c}} & =\left(-\frac{1}{2},-\frac{\sqrt{3}}{2}\right)-\left(\frac{\Gamma_{1}}{\Gamma}-\frac{\Gamma_{1}+\Gamma_{2}}{2 \Gamma}, \frac{\sqrt{3}}{2 \Gamma}\left(\Gamma_{2}-\Gamma_{3}\right)\right) \\
& =\left(-\frac{1}{2}-\frac{\Gamma_{1}}{\Gamma}+\frac{\Gamma_{1}+\Gamma_{2}}{2 \Gamma},-\frac{\sqrt{3}}{2}-\frac{\sqrt{3}}{2 \Gamma}\left(\Gamma_{2}-\Gamma_{3}\right)\right) \\
& =\left(-\frac{3 \Gamma_{1}}{2 \Gamma},-\frac{\sqrt{3}}{2 \Gamma}\left(\Gamma_{1}+2 \Gamma_{2}\right)\right) .
\end{aligned}
$$

Summing up all computations we get

$$
\begin{aligned}
& \left\langle M_{\Gamma} \boldsymbol{\xi}, \boldsymbol{\xi}\right\rangle=\left[\begin{array}{c}
\frac{\Gamma_{1}}{\Gamma}-\frac{\Gamma_{1}+\Gamma_{2}}{2 \Gamma} \\
\frac{\sqrt{3}}{2 \Gamma}\left(\Gamma_{2}-\Gamma_{3}\right) \\
-\frac{3 \Gamma_{1}}{2 \Gamma} \\
\frac{\sqrt{3}}{2 \Gamma}\left(\Gamma_{1}+2 \Gamma_{3}\right) \\
-\frac{3 \Gamma_{1}}{2 \Gamma} \\
-\frac{\sqrt{3}}{2 \Gamma}\left(\Gamma_{1}+2 \Gamma_{2}\right)
\end{array}\right] \quad\left[\begin{array}{cccccc}
\Gamma_{1} & 0 & 0 & 0 & 0 & 0 \\
0 & \Gamma_{1} & 0 & 0 & 0 & 0 \\
0 & 0 & \Gamma_{2} & 0 & 0 & 0 \\
0 & 0 & 0 & \Gamma_{2} & 0 & 0 \\
0 & 0 & 0 & 0 & \Gamma_{3} & 0 \\
0 & 0 & 0 & 0 & 0 & \Gamma_{3}
\end{array}\right] \cdot\left[\begin{array}{c}
\frac{\Gamma_{1}}{\Gamma}-\frac{\Gamma_{1}+\Gamma_{2}}{2 \Gamma} \\
\frac{\sqrt{3}}{2 \Gamma}\left(\Gamma_{2}-\Gamma_{3}\right) \\
-\frac{3 \Gamma_{1}}{2 \Gamma} \\
\frac{\sqrt{3}}{2 \Gamma}\left(\Gamma_{1}+2 \Gamma_{3}\right) \\
-\frac{3 \Gamma_{1}}{2 \Gamma} \\
\sqrt{3} \\
-\frac{\sqrt{2}}{2 \Gamma}\left(\Gamma_{1}+2 \Gamma_{2}\right)
\end{array}\right] \\
& =\frac{\left(3 \Gamma_{2}+3 \Gamma_{3}\right)^{2} \Gamma_{1}}{4 \Gamma^{2}}+\frac{3\left(\Gamma_{2}-\Gamma_{3}\right)^{2} \Gamma_{1}}{4 \Gamma^{2}}+\frac{9 \Gamma_{1}^{2} \Gamma_{2}}{4 \Gamma^{2}}+\frac{3\left(\Gamma_{1}+2 \Gamma_{3}\right)^{2} \Gamma_{2}}{4 \Gamma^{2}} \\
& +\frac{9 \Gamma_{1}^{2} \Gamma_{3}}{4 \Gamma^{2}}+\frac{3\left(\Gamma_{1}+2 \Gamma_{2}\right)^{2} \Gamma_{3}}{4 \Gamma^{2}} \\
& =\frac{3}{4 \Gamma^{2}}\left(3\left(\Gamma_{2}+\Gamma_{3}\right)^{2} \Gamma_{1}+\left(\Gamma_{2}-\Gamma_{3}\right)^{2} \Gamma_{1}+3 \Gamma_{1}^{2} \Gamma_{2}+\left(\Gamma_{1}+2 \Gamma_{3}\right)^{2} \Gamma_{2}\right. \\
& \left.+3 \Gamma_{1}^{2} \Gamma_{3}+\left(\Gamma_{1}+2 \Gamma_{2}\right)^{2} \Gamma_{3}\right) \\
& =\frac{3}{\Gamma^{2}}\left(\Gamma\left(\Gamma_{1}^{2}+\Gamma_{2}^{2}+\Gamma_{3}^{2}\right)+3 \Gamma_{1} \Gamma_{2} \Gamma_{3}\right) \text {. }
\end{aligned}
$$

Let $a:=\Gamma\left(\Gamma_{1}^{2}+\Gamma_{2}^{2}+\Gamma_{3}^{2}\right)+3 \Gamma_{1} \Gamma_{2} \Gamma_{3}$, then the signature of the quadratic form $\left\langle M_{\Gamma} \xi, \xi\right\rangle$ coincides with that of the quadratic form $a$.

We distinguish the following four cases.

First case. We assume that $\Gamma_{1}, \Gamma_{2}, \Gamma_{3}>0$. In this case we have $\omega>0$ and $\left\langle M_{\Gamma} \xi, \xi\right\rangle>$ 0 and so, according to Theorem 2 , we get that $\mathrm{n}_{-}\left(\widehat{A}_{\Gamma}\right)=0$. 
Second case. We assume that $\Gamma_{1}, \Gamma_{2}, \Gamma_{3}<0$. Thus we have $\omega<0$ and $\left\langle M_{\Gamma} \xi, \xi\right\rangle<0$; so, according to Theorem 2 , we get that $\mathrm{n}_{-}\left(\widehat{A}_{\Gamma}\right)=0$.

Third case. We assume that $\Gamma_{i}<0$ only for one index $i$. In this case, without losing in generalities, we may assume that $\Gamma_{3}<0$. Since $L>0$, then we have that

$$
-\frac{\Gamma_{1} \Gamma_{2}}{\Gamma_{1}+\Gamma_{2}}<\Gamma_{3}<0
$$

We claim that $\left\langle M_{\Gamma} \xi, \xi\right\rangle>0$. In order to prove this, as already observed, it is enough to prove that $a>0$. We observe that $a$ could be re-written as follows

$$
\begin{aligned}
a & =\Gamma\left(\Gamma_{1}^{2}+\Gamma_{2}^{2}+\Gamma_{3}^{2}\right)+3 \Gamma_{1} \Gamma_{2} \Gamma_{3} \\
& =\left(\Gamma_{1}+\Gamma_{2}+\Gamma_{3}\right)\left(\Gamma_{1}^{2}+\Gamma_{2}^{2}+\Gamma_{3}^{2}\right)+3 \Gamma_{1} \Gamma_{2} \Gamma_{3} \\
& =\Gamma_{1}^{3}\left(\left(1+\frac{\Gamma_{2}}{\Gamma_{1}}+\frac{\Gamma_{3}}{\Gamma_{1}}\right)\left(1+\frac{\Gamma_{2}^{2}}{\Gamma_{1}^{2}}+\frac{\Gamma_{3}^{2}}{\Gamma_{1}^{2}}\right)+3 \frac{\Gamma_{2} \Gamma_{3}}{\Gamma_{1}^{2}}\right) .
\end{aligned}
$$

We let

$$
x=\frac{\Gamma_{2}}{\Gamma_{1}}, y=\frac{\Gamma_{3}}{\Gamma_{1}} .
$$

Since $\Gamma_{1}>0$, Thus the signature of the quadratic form $a$ agrees with the sign of the function $b$ defined below

$$
b(x, y):=(1+x+y)\left(1+x^{2}+y^{2}\right)+3 x y,
$$

where $x>0,-\frac{x}{1+x}<y<0$.

Fix $x \in[0,+\infty)$, differentiating $(1+x+y)\left(1+x^{2}+y^{2}\right)+3 x y$ with respect to $y$, yields

$$
3 y^{2}+2 y(x+1)+3 x+x^{2}+1=3\left(y+\frac{x+1}{3}\right)^{2}+\frac{2 x^{2}+7 x+2}{3}>0 .
$$

This implies that $y \mapsto b(x, y)$ is a monotone increasing function (with respect to $y$ ) thus the infimum is getting precisely at $-\frac{x}{1+x}$. Setting $y=-\frac{x}{1+x}$, then we get

$$
\begin{aligned}
& \left(1+x-\frac{x}{1+x}\right)\left(1+x^{2}+\frac{x^{2}}{(1+x)^{2}}\right)-3 \frac{x^{2}}{1+x} \\
& =\frac{1}{(1+x)^{3}}\left(x^{6}+3 x^{5}+3 x^{4}+x^{3}+3 x^{2}+3 x+1\right)>0 \quad \forall x \in[0,+\infty) .
\end{aligned}
$$


By this, we immediately get that for every $x \in(0,+\infty)$ and $-\frac{x}{1+x}<y<0$, the function $b$ is positive or which is the same that the quadratic form $a$ is positive definite, hence $\left\langle M_{\Gamma} \boldsymbol{\xi}, \boldsymbol{\xi}\right\rangle>0$.

As direct consequence of Eq. (2.9) as well as of the fact that $L>0$ and $\left\langle M_{\Gamma} \xi, \xi\right\rangle>$ 0 , we get that $\omega>0$. So now, according to Theorem 2 , we have that $\mathrm{n}_{-}\left(\widehat{A}_{\Gamma}\right)=$ $\mathrm{n}_{-}\left(M_{\Gamma}\right)-1=1$.

Fourth case. We assume now that $\Gamma_{i}>0$ only for one index $i$. Arguing precisely as before we get $\left\langle M_{\Gamma} \xi, \xi\right\rangle<0$. In this case, however as direct consequence of Eq. (2.9) s well as of the fact that $L>0$ and $\left\langle M_{\Gamma} \boldsymbol{\xi}, \boldsymbol{\xi}\right\rangle<0$, we get that $\omega<0$. According to Theorem 2, we have that $\mathrm{n}_{-}\left(\widehat{A}_{\Gamma}\right)=\mathrm{n}_{+}\left(M_{\Gamma}\right)=2$.

\subsection{The Rhombus Families}

From paper [7], we know that there exist two families of relative equilibria where the configuration is a rhombus. Set $\Gamma_{1}=\Gamma_{2}=1$ and $\Gamma_{3}=\Gamma_{4}=m$, where $m \in(-1,1]$ is a parameter. Place the vortices at $z_{1}=(1,0), z_{2}=(-1,0), z_{3}=(0, y)$ and $z_{4}=(0,-y)$, forming a rhombus with diagonals lying on the coordinate axis. This configuration is a central configuration provided that

$$
y^{2}=\frac{1}{2}\left(\beta \pm \sqrt{\beta^{2}+4 m}\right), \quad \beta=3(1-m),
$$

or, equivalently,

$$
m=\frac{3 y^{2}-y^{4}}{3 y^{2}-1} .
$$

The angular velocity is given by

$$
\omega=\frac{m^{2}+4 m+1}{2\left(1+m y^{2}\right)}=\frac{1}{2}+\frac{2 m}{y^{2}+1} .
$$

The case $m<-1$ or $m>1$ can be reduced to this setup through a rescaling of the circulations and a relabeling of the vortices. There are two solutions depending on the sign choice for $y^{2}$.

Taking plus sign in Eq. (5.1) yields a solution for $m \in(-1,1]$ that always has $\omega>0$. We will call this solution rhombus $A$. Taking - in Eq. (5.1) yields a solution for $m \in(-1,0)$ having $\omega>0$ for $m \in(-2+\sqrt{3}, 0)$, but $\omega<0$ for $m \in(-1,-2+\sqrt{3})$. We will call this solution rhombus $B$.

In the aforementioned paper, author computed the nontrivial eigenvectors of $M_{\Gamma}^{-1} D^{2} H(\xi)$; in particular he proved that they are reals for every value of $m$ and are given by

$$
\begin{aligned}
& \boldsymbol{v}_{1}=[m y, 0,-m y, 0,0,-1,0,1]^{\top} \text { and } K \boldsymbol{v}_{1} \\
& \boldsymbol{v}_{2}=[m, 0, m, 0,-1,0,-1,0]^{\top} \quad \text { and } K \boldsymbol{v}_{2} .
\end{aligned}
$$


Theorem 5.2 ([7, Theorem 4.1]) Under the previous notation, the following holds.

1. Rhombus $A$ is linearly stable for $-2+\sqrt{3}<m \leq 1$. At $m=-2+\sqrt{3}$ the relative equilibrium is degenerate. For $-1<m<-2+\sqrt{3}$, rhombus $A$ is unstable and the nontrivial eigenvalues consist of a real pair and a pure imaginary pair.

2. Rhombus B is always unstable. One pair of eigenvalues is always real. The other pair of eigenvalues is purely imaginary for $-1<m<m^{*}$ and real for $m^{*}<m<$ 0 , where $m^{*}$ is the only real root of the cubic $9 m^{3}+3 m^{2}+7 m+5$. At $m=m^{*}$, rhombus $B$ is degenerate.

As consequence of Theorems 2 and 5.2, we get information on the Morse index of the rhombi configurations.

Theorem 5.3 We assume that $z$ is the relative equilibrium generated by the rhombus central configuration $\xi$. Then

1. if the central configuration corresponds to rhombus A, then we have

$$
\mathrm{n}_{-}\left(\widehat{A}_{\Gamma}(\xi)\right)=\left\{\begin{array}{lll}
0 & \text { if } & 0<m \leq 1, \\
3 & \text { if } \quad-2+\sqrt{3}<m<0, \\
4 & \text { if } \quad-1<m<-2+\sqrt{3}
\end{array}\right.
$$

2. if the central configuration corresponds to rhombus $B$, then we have

$$
\mathrm{n}_{-}\left(\widehat{A}_{\Gamma}(\xi)\right)=\left\{\begin{array}{lll}
2 & \text { if } & -2+\sqrt{3}<m<0, \\
4 & \text { if } & m^{*}<m<-2+\sqrt{3}, \\
3 & \text { if } & -1<m<m^{*}
\end{array}\right.
$$

where $m^{*}$ is the only real root of the cubic $9 m^{3}+3 m^{2}+7 m+5$.

Proof In order to prove the first claim, we observe that, by the computation performed by author in [7, p. 1129] as well as consequence of Lemmas 2.15 and 3.3, it follows that the spectrum of the matrix $A_{\Gamma}$ is given by

$$
\begin{aligned}
& \sigma\left(A_{\Gamma}\right)=\left\{0,2 \omega, \omega, \omega-\mu_{1}, \omega+\mu_{1}, \omega-\mu_{2}, \omega+\mu_{2}\right\} \text { where } \\
& \mu_{1}=\frac{7 y^{4}-18 y^{2}+7}{2\left(y^{2}+1\right)\left(3 y^{2}-1\right)} \text { and } \mu_{2}=\frac{2(m+1)\left(1-y^{2}\right)}{\left(1+y^{2}\right)^{2}} \\
& \quad=\frac{2\left(y^{2}-1\right)\left(y^{2}+2 y-1\right)\left(y^{2}-2 y-1\right)}{\left(y^{2}+1\right)^{2}\left(3 y^{2}-1\right)}
\end{aligned}
$$

where the algebraic multiplicity of $\omega$ is two. Moreover

$$
\begin{aligned}
& A_{\Gamma} \boldsymbol{\xi}=0, \quad A_{\Gamma} K \boldsymbol{\xi}=2 \omega K \boldsymbol{\xi}, \quad A_{\Gamma} \boldsymbol{s}=\omega \boldsymbol{s}, \quad A_{\Gamma} K \boldsymbol{s}=\omega \boldsymbol{s}, \\
& A_{\Gamma} \boldsymbol{v}_{1}=\left(\mu_{1}+\omega\right) \boldsymbol{v}_{1}, \quad A_{\Gamma} K \boldsymbol{v}_{1}=\left(-\mu_{1}+\omega\right) K \boldsymbol{v}_{1}, \\
& A_{\Gamma} \boldsymbol{v}_{2}=\left(\mu_{2}+\omega\right) \boldsymbol{v}_{2}, \quad A_{\Gamma} K \boldsymbol{v}_{2}=\left(-\mu_{2}+\omega\right) K \boldsymbol{v}_{2}
\end{aligned}
$$


where $s$ is the vector defined in Lemma 2.15. Thus, by using Lemma 3.9, we get the following $M_{\Gamma^{-}}$-orthogonal direct sum decomposition

$$
\mathbb{C}^{8}=I_{0} \oplus I_{2 \omega} \oplus I_{\omega} \oplus I_{-\mu_{1}+\omega} \oplus I_{\mu_{1}+\omega} \oplus I_{\mu_{2}+\omega} \oplus I_{-\mu_{2}+\omega}
$$

where

$$
\begin{aligned}
& I_{0}=\operatorname{span}\{\boldsymbol{\xi}\}, I_{2 \omega}=\operatorname{span}\{K \boldsymbol{\xi}\}, I_{\omega}=\operatorname{span}\{\boldsymbol{s}, K \boldsymbol{s}\}, I_{\omega+\mu_{1}}=\operatorname{span}\left\{\boldsymbol{v}_{1}\right\} \\
& I_{\omega-\mu_{1}}=\operatorname{span}\left\{K \boldsymbol{v}_{1}\right\}, I_{\omega+\mu_{2}}=\operatorname{span}\left\{\boldsymbol{v}_{2}\right\}, I_{\omega-\mu_{1}}=\operatorname{span}\left\{K \boldsymbol{v}_{2}\right\}
\end{aligned}
$$

By a straightforward calculations, we get that

$$
\begin{aligned}
& \left.\widehat{A_{\Gamma}}\right|_{I_{0}}[\boldsymbol{\xi}]=\left\langle M_{\Gamma} A_{\Gamma} \boldsymbol{\xi}, \boldsymbol{\xi}\right\rangle=0,\left.\quad \widehat{A_{\Gamma}}\right|_{I_{2 \omega}}[K \boldsymbol{\xi}]=\left\langle M_{\Gamma} A_{\Gamma} K \boldsymbol{\xi}, K \boldsymbol{\xi}\right\rangle=2 \omega\left\langle M_{\Gamma} \boldsymbol{\xi}, \boldsymbol{\xi}\right\rangle \\
& \left.\widehat{A_{\Gamma}}\right|_{I_{\omega}}[\boldsymbol{s}, K \boldsymbol{s}]=\left[\begin{array}{cc}
\left\langle M_{\Gamma} A_{\Gamma} \boldsymbol{s}, \boldsymbol{s}\right\rangle & \left\langle M_{\Gamma} A_{\Gamma} \boldsymbol{s}, K \boldsymbol{s}\right\rangle \\
\left\langle M_{\Gamma} A_{\Gamma} K \boldsymbol{s}, \boldsymbol{s}\right\rangle & \left\langle M_{\Gamma} A_{\Gamma} K \boldsymbol{s}, K \boldsymbol{s}\right\rangle
\end{array}\right]=\left[\begin{array}{cc}
\omega\left\langle M_{\Gamma} \boldsymbol{s}, \boldsymbol{s}\right) & \omega\left(M_{\Gamma} \boldsymbol{s}, K \boldsymbol{s}\right\rangle \\
\omega\left(M_{\Gamma} K \boldsymbol{s}, \boldsymbol{s}\right) & \omega\left\langle M_{\Gamma} \boldsymbol{s}, \boldsymbol{s}\right\rangle
\end{array}\right] \\
& =\omega\left[\begin{array}{cc}
2+m & 0 \\
0 & 2+m
\end{array}\right],\left.\widehat{A_{\Gamma}}\right|_{I_{\omega+\mu_{1}}}\left[\boldsymbol{v}_{1}\right]=\left\langle M_{\Gamma} A_{\Gamma} \boldsymbol{v}_{1}, \boldsymbol{v}_{1}\right\rangle=\left(\omega+\mu_{1}\right)\left\langle M_{\Gamma} \boldsymbol{v}_{1}, \boldsymbol{v}_{1}\right\rangle \\
& \left.\widehat{A_{\Gamma}}\right|_{I_{\omega-\mu_{1}}}\left[\boldsymbol{v}_{1}\right]=\left\langle M_{\Gamma} A_{\Gamma} K \boldsymbol{v}_{1}, K \boldsymbol{v}_{1}\right\rangle=\left(\omega-\mu_{1}\right)\left\langle M_{\Gamma} \boldsymbol{v}_{1}, \boldsymbol{v}_{1}\right\rangle, \\
& \left.\widehat{A_{\Gamma}}\right|_{I_{\omega+\mu_{2}}}\left[\boldsymbol{v}_{2}\right]=\left\langle M_{\Gamma} A_{\Gamma} \boldsymbol{v}_{2}, \boldsymbol{v}_{2}\right\rangle=\left(\omega+\mu_{2}\right)\left\langle M_{\Gamma} \boldsymbol{v}_{2}, v\right\rangle \\
& \left.\left.\widehat{A_{\Gamma}}\right|_{I_{\omega-\mu_{2}}}\left[\boldsymbol{v}_{2}\right]=\left\langle M_{\Gamma} A_{\Gamma} K \boldsymbol{v}_{2}, K \boldsymbol{v}_{2}\right\rangle\right)=\left(\omega-\mu_{2}\right)\left\langle M_{\Gamma} \boldsymbol{v}_{2}, \boldsymbol{v}_{2}\right\rangle .
\end{aligned}
$$

By the rhombi classifications, we distinguish the two cases.

Rhombus A central configuration. We assume that the central configuration corresponds to rhombus $A$. Then, as already observed, we have

$$
y^{2}=\frac{1}{2}\left(3(1-m)+\sqrt{9(1-m)^{2}+4 m}\right)
$$

and $\omega$ is always positive for all $m \in(-1,1]$. Since $M_{\Gamma}$ is the diagonal block matrix given by $M_{\Gamma}=\operatorname{diag}\left(I_{4}, m I_{4}\right)$ and the central configuration $\xi=$ $[1,0,-1-0,0, y, 0,-y]^{\top}$, we immediately get that

$$
\left\langle M_{\Gamma} \boldsymbol{\xi}, \boldsymbol{\xi}\right\rangle=3 m-3 m^{2}+m \sqrt{9(1-m)^{2}+4 m}+2
$$

Define the function $r(m):=3 m-3 m^{2}+m \sqrt{9(1-m)^{2}+4 m}+2$ and in order to find the root of the equation $r(m)=0$, we first compute the roots of the equation

$$
\left(3 m-3 m^{2}+2\right)^{2}-\left(m \sqrt{9(1-m)^{2}+4 m}\right)^{2}=0 .
$$


By a simple calculation we get $\mathrm{s}$ that

$$
\begin{aligned}
& \left(3 m-3 m^{2}+2\right)^{2}-\left(m \sqrt{9(1-m)^{2}+4 m}\right)^{2} \\
& =9 m^{4}-18 m^{3}-3 m^{2}+12 m+4-\left(9 m^{4}-14 m^{3}+9 m^{2}\right) \\
& =-4 m^{3}-12 m^{3}+12 m^{2}+4=-4(m-1)(m+2+\sqrt{3})(m+2-\sqrt{3}) .
\end{aligned}
$$

This implies that $\left(3 m-3 m^{2}+2\right)^{2}-\left(m \sqrt{9(1-m)^{2}+4 m}\right)^{2}=0$ has three real roots given by $-2-\sqrt{3},-2+\sqrt{3}$ and 1 . Moreover, $r(1)=4>0, r(-2+\sqrt{3})=0$ and $r(2+\sqrt{3})=-50-30 \sqrt{3}<0$, and then the function $m \mapsto r(m)$ has the unique root given by $-2+\sqrt{3}$. So we have that

$$
\begin{cases}\left\langle M_{\Gamma} \boldsymbol{\xi}, \boldsymbol{\xi}\right\rangle>0, & \text { if }-2+\sqrt{3}<m \leq 1 \\ \left\langle M_{\Gamma} \boldsymbol{\xi}, \boldsymbol{\xi}\right\rangle<0, & \text { if }-2-\sqrt{3}<m<-2+\sqrt{3}\end{cases}
$$

- Linearly stable relative equilibria. If $-2+\sqrt{3}<m \leq 1$ directly by Theorem 5.2 we get that $z$ is linearly stable. So by Theorem 2, by Eq. (5.5) and by remembering that fin this case the angular velocity $\omega$ is always positive we conclude that

$$
\mathrm{n}_{-}\left(\widehat{A}_{\Gamma}\right)= \begin{cases}n_{-}\left(M_{\Gamma}\right)=0 & \text { if } 0<m \leq 1 \\ n_{-}\left(M_{\Gamma}\right)-1=3 & \text { if }-2+\sqrt{3}<m<0 .\end{cases}
$$

- Unstable relative equilibria. By invoking once again the first claim in Theorem 5.2, we know that if $-1<m<-2+\sqrt{3}$, then the relative equilibrium $z$ is unstable and the nontrivial eigenvalues consist of a pair of real and a pair of purely imaginary eigenvalues. By Lemma 3.2 it follows that the non-trivial eigenvalues of $B$ are $\pm \sqrt{-\omega^{2}+\mu_{1}^{2}}$ and $\pm \sqrt{-\omega^{2}+\mu_{2}^{2}}$. Moreover, for $-1<m<-2+\sqrt{3}$, author in [7] proved that $\sqrt{-\omega^{2}+\mu_{1}^{2}} \in \mathbb{R}$ and $\sqrt{-\omega^{2}+\mu_{2}^{2}} \in i \mathbb{R}$. Summing up, we get that $\left|\mu_{1}\right|>\omega$ whilst $\left|\mu_{2}\right|<\omega$.

Being $\left|\mu_{1}\right|>\omega$, we have that $\left(\omega+\mu_{1}\right)\left(\omega-\mu_{1}\right)<0$ for $m \in(-1,-2+\sqrt{3})$ which means that the factors $\omega \pm \mu_{1}$ have opposite signs. By Eq. (5.4)

$$
\left.\widehat{A_{\Gamma}}\right|_{I_{\omega+\mu_{1}}}\left[\boldsymbol{v}_{1}\right]=\left(\omega+\mu_{1}\right)\left\langle M_{\Gamma} \boldsymbol{v}_{1}, \boldsymbol{v}_{1}\right\rangle \quad \text { and }\left.\quad \widehat{A_{\Gamma}}\right|_{I_{\omega-\mu_{1}}}\left[\boldsymbol{v}_{1}\right]=\left(\omega-\mu_{1}\right)\left\langle M_{\Gamma} \boldsymbol{v}_{1}, \boldsymbol{v}_{1}\right\rangle
$$

Each of the forms $\left.\widehat{A_{\Gamma}}\right|_{I_{\omega \pm \mu_{1}}}$ is a quadratic form onto a one-dimensional space and by the previous discussion on signs, we get that $\mathrm{n}_{-}\left(\left.\widehat{A_{\Gamma}}\right|_{I_{\omega+\mu_{1}}}\right)+$ $\mathrm{n}_{-}\left(\left.\widehat{A_{\Gamma}}\right|_{I_{\omega-\mu_{1}}}\right)=1$ for $m \in(-1,-2+\sqrt{3})$. By a straightforward calculation, we also get that $\left\langle M_{\Gamma} \boldsymbol{v}_{2}, \boldsymbol{v}_{2}\right\rangle=2 m^{2}+2 m$ where $\boldsymbol{v}_{2}$ was given in Eq. (5.2). We observe that $\left\langle M_{\Gamma} \boldsymbol{v}_{2}, \boldsymbol{v}_{2}\right\rangle$ is negative for $m \in(-1,0)$ and a fortiori for $m \in(-1,-2+\sqrt{3})$. 
Now since $\left|\mu_{2}\right|<\omega$, this implies that $\left(\omega+\mu_{2}\right)\left(\omega-\mu_{2}\right)>0$. However, by the definition of $\mu_{2}$ given in Eq. (5.3), we infer that $\mu_{2}<0$ and in particular being $\omega>0$ we get that $\omega-\mu_{2}>0$. Thus by the product rule it then follows that also $\omega+\mu_{2}>0$ Since,

$$
\left.\widehat{A_{\Gamma}}\right|_{I_{\omega+\mu_{2}}}\left[\boldsymbol{v}_{2}\right]=\left(\omega+\mu_{2}\right)\left\langle M_{\Gamma} \boldsymbol{v}_{2}, \boldsymbol{v}_{2}\right\rangle \quad \text { and }\left.\quad \widehat{A_{\Gamma}}\right|_{I_{\omega-\mu_{2}}}\left[\boldsymbol{v}_{2}\right]=\left(\omega-\mu_{2}\right)\left\langle M_{\Gamma} \boldsymbol{v}_{2}, \boldsymbol{v}_{2}\right\rangle
$$

we finally get both quadratic forms (on the one-dimensional subspace generated by $\boldsymbol{v}_{2}$ ) are negative definite and hence each gives a 1 contribution to the Morse index. In conclusion, we get

$$
\begin{aligned}
\mathrm{n}_{-}\left(\widehat{A_{\Gamma}}\right)= & \mathrm{n}_{-}\left(\left.\widehat{A_{\Gamma}}\right|_{I_{0}}\right)+\mathrm{n}_{-}\left(\left.\widehat{A_{\Gamma}}\right|_{I_{2 \omega}}\right)+\mathrm{n}_{-}\left(\left.\widehat{A_{\Gamma}}\right|_{I_{\omega}}\right)+\mathrm{n}_{-}\left(\left.\widehat{A_{\Gamma}}\right|_{I_{\omega+\mu_{1}}}\right) \\
& +\mathrm{n}_{-}\left(\left.\widehat{A}\right|_{I_{\omega-\mu_{1}}}\right)+\mathrm{n}_{-}\left(\left.\widehat{A}\right|_{I_{\omega+\mu_{2}}}\right)+\mathrm{n}_{-}\left(\left.\widehat{A}\right|_{I_{\omega-\mu_{2}}}\right) \\
= & 0+1+0+1+1+1 \\
= & 4 .
\end{aligned}
$$

Rhombus $B$ central configuration. We assume now that the relative equilibrium $z_{0}$ comes out from rhombus B central configuration. Once again by using Theorem 5.2, it follows that $z_{0}$ is always unstable for $m \in(-1,0)$, moreover, we know that

$$
\left\{\begin{array}{lll}
\omega>0 & \text { if } & m \in(-2+\sqrt{3}, 0) \\
\omega<0 & \text { if } & m \in(-1,-2+\sqrt{3}) .
\end{array}\right.
$$

and one pair of eigenvalues is always real whilst the other is purely imaginary for $-1<m<m^{*}$ and real for $m^{*}<m<0$, where $m^{*} \approx-0.5951$ is the only real root of the cubic $9 m^{3}+3 m^{2}+7 m+5$. At $m=m^{*}$, rhombus $B$ is degenerate.

By invoking Lemma 3.2, the non-trivial eigenvalues of $B$ are $\pm \sqrt{-\omega^{2}+\mu_{1}^{2}}$ and $\pm \sqrt{-\omega^{2}+\mu_{2}^{2}}$. Furthermore by [7, p. 1129], we know also that

$$
\begin{cases}\sqrt{-\omega^{2}+\mu_{1}^{2}} & \text { is real if } m \in(-1,0), \\ \sqrt{-\omega^{2}+\mu_{2}^{2}} & \text { is real if } m \in\left(m^{*}, 0\right), \\ \sqrt{-\omega^{2}+\mu_{2}^{2}} & \text { is purely imaginary if } m \in\left(-1, m^{*}\right) .\end{cases}
$$

Thus we get

$$
\begin{cases}\left|\mu_{1}\right|>|\omega|, & \text { if } m \in(-1,0) \\ \left|\mu_{2}\right|>|\omega|, & \text { if } m \in\left(m^{*}, 0\right) \\ \left|\mu_{2}\right|<|\omega|, & \text { if } m \in\left(-1, m^{*}\right)\end{cases}
$$


Being $\left|\mu_{1}\right|>|\omega|$, we have that $\left(\omega+\mu_{1}\right)\left(\omega-\mu_{1}\right)<0$ for $m \in(-1,0)$. By Eq. (5.4)

$\left.\widehat{A_{\Gamma}}\right|_{I_{\omega+\mu_{1}}}\left[\boldsymbol{v}_{1}\right]=\left(\omega+\mu_{1}\right)\left\langle M_{\Gamma} \boldsymbol{v}_{1}, \boldsymbol{v}_{1}\right\rangle \quad$ and $\left.\quad \widehat{A_{\Gamma}}\right|_{I_{\omega-\mu_{1}}}\left[\boldsymbol{v}_{1}\right]=\left(\omega-\mu_{1}\right)\left\langle M_{\Gamma} \boldsymbol{v}_{1}, \boldsymbol{v}_{1}\right\rangle$

then we can conclude that $\mathrm{n}_{-}\left(\left.\widehat{A_{\Gamma}}\right|_{I_{\omega+\mu_{1}}}\right)+\mathrm{n}_{-}\left(\left.\widehat{A_{\Gamma}}\right|_{I_{\omega-\mu_{1}}}\right)=1$. By the very some argument, we infer also that $\mathrm{n}_{-}\left(\left.\widehat{A_{\Gamma}}\right|_{I_{\omega+\mu_{2}}}\right)+\mathrm{n}_{-}\left(\left.\widehat{A_{\Gamma}}\right|_{I_{\omega-\mu_{2}}}\right)=1$ for $m \in\left(m^{*}, 0\right)$. Next, we will compute the sign of $\left.\widehat{A_{\Gamma}}\right|_{I_{\omega \pm \mu_{2}}}$ for $m \in\left(-1, m^{*}\right)$. Since $\left\langle M_{\Gamma} \boldsymbol{v}_{2}, \boldsymbol{v}_{2}\right\rangle=$ $2 m^{2}+2 m$ which is negative in $(-1,0)$, a fortiori it will be negative in for $m \in\left(-1, m^{*}\right)$. Since $-2+\sqrt{3} \approx-0.2679>-0.5951 \approx m^{*}$, then $\omega<0$ for $\left(-1, m^{*}\right)$. Now, since $-\omega^{2}+\mu_{2}^{2}<0$ we get that $\left(\mu_{2}-\omega\right)\left(\mu_{2}+\omega\right)<0$ and since $\mu_{2}+\omega<0$ (in fact $\omega$ is negative for $m \in\left(-1, m^{*}\right)$ as well as $\left.\mu_{2}\right)$, this implies that $\mu_{2}-\omega>0$. Thus in conclusion both eigenvalues $\mu_{2} \pm \omega$ are negative in $\left(-1, m^{*}\right)$. By Eq. (5.4), we have that

$$
\left.\widehat{A_{\Gamma}}\right|_{I_{\omega+\mu_{2}}}\left[\boldsymbol{v}_{2}\right]=\left(\omega+\mu_{2}\right)\left\langle M_{\Gamma} \boldsymbol{v}_{2}, \boldsymbol{v}_{2}\right\rangle \quad \text { and }\left.\quad \widehat{A_{\Gamma}}\right|_{I_{\omega-\mu_{2}}}\left[\boldsymbol{v}_{2}\right]=\left(\omega-\mu_{2}\right)\left\langle M_{\Gamma} \boldsymbol{v}_{2}, \boldsymbol{v}_{2}\right\rangle
$$

and immediately by the discussion above, we conclude that

$$
\mathrm{n}_{-}\left(\left.\widehat{A_{\Gamma}}\right|_{I_{\omega+\mu_{2}}}\right)=n_{-}\left(\left.\widehat{A_{\Gamma}}\right|_{I_{\omega-\mu_{2}}}\right)=0 \text { for } \quad m \in\left(-1, m^{*}\right)
$$

Invoking once again Eq. (5.4), we get that $\left.\widehat{A_{\Gamma}}\right|_{I_{2 \omega}}[K \boldsymbol{\xi}]=2 \omega\left\langle M_{\Gamma} K \boldsymbol{\xi}\right.$, $\left.\boldsymbol{\xi}\right\rangle$. Summing up Eqs. (5.5) and (5.6), then we get

$$
\mathrm{n}_{-}\left(\left.\widehat{A_{\Gamma}}\right|_{I_{2 \omega}}\right)=0 \text { for all } m \in(-1,-2+\sqrt{3}) \cup(-2+\sqrt{3}, 0)
$$

By Eq. (5.4), we get finally that

$$
\mathrm{n}_{-}\left(\widehat{A_{\Gamma}}\right)= \begin{cases}1+1=2 & \text { if } \quad-2+\sqrt{3}<m<0 \\ 2+1+1=4 & \text { if } \quad m^{*}<m<-2+\sqrt{3} \\ 2+1=3 & \text { if } \quad-1<m<m^{*}\end{cases}
$$

This concludes the proof.

Acknowledgements Open access funding provided by Universitá degli Studi di Torino within the CRUICARE Agreement. The second named author wishes to thank all faculties and staff of the Mathematics Department in the Shandong University (Jinan) for providing excellent working conditions during his stay. We would like to warmly thanks Gareth Roberts for several fruitful and interesting discussions on this research project. We finally thank the anonymous referee for reading the paper carefully and providing thoughtful comments. 
Open Access This article is licensed under a Creative Commons Attribution 4.0 International License, which permits use, sharing, adaptation, distribution and reproduction in any medium or format, as long as you give appropriate credit to the original author(s) and the source, provide a link to the Creative Commons licence, and indicate if changes were made. The images or other third party material in this article are included in the article's Creative Commons licence, unless indicated otherwise in a credit line to the material. If material is not included in the article's Creative Commons licence and your intended use is not permitted by statutory regulation or exceeds the permitted use, you will need to obtain permission directly from the copyright holder. To view a copy of this licence, visit http://creativecommons.org/licenses/by/4.0/.

\section{References}

1. von Helmholtz, H.: Über integrale der hydrodynamischen Gleichungen, welcheden Wirbelbewegungen entsprechen. J. Math. Bd. LV. Heft 1, 4 (1858)

2. Routh, E.J.: Some applications of conjugate functions. Proc. Lond. Math. Soc. 1(1), $73-89$ (1880)

3. Lim, C.C.: On the Motion of Vortices in Two Dimensions. University of Toronto Press, Toronto (1943)

4. Newton, P.K.: The N-vortex Problem. Analytical Techniques. Applied Mathematical Sciences, vol. 145. Springer, New York (2001)

5. Hu, X., Long, Y., Sun, S.: Linear stability of elliptic Lagrangian solutions of the planar three-body problem via index theory. Arch. Ration. Mech. Anal. 213(3), 993-1045 (2014)

6. Barutello, V., Jadanza, R.D., Portaluri, A.: Morse index and linear stability of the Lagrangian circular orbit in a three-body-type problem via index theory. Arch. Ration. Mech. Anal. 219(1), 387-444 (2016)

7. Roberts, G.E.: Stability of relative equilibria in the planar N-vortex problem. SIAM J. Appl. Dyn. Syst. 12, 1114-1134 (2013)

8. Roberts, G.E.: Morse theory and relative equilibria in the planar n-vortex problem. Arch. Ration. Mech. Anal. 228(1), 209-236 (2018)

9. Synge, J.L.: On the motion of three vortices. Can. J. Math. 1, 257-270 (1949)

10. Palais, R.S.: The principle of symmetric criticality. Commun. Math. Phys. 69, 19-30 (1979)

11. Gohberg, I., Lancaster, P., Rodman, L.I.: Linear Algebra and Applications. Birkhäuser Verlag, Basel (2005)

12. Palmore, J.: Relative equilibria of vortices in two dimensions. Proc. Natl. Acad. Sci. USA 79, 716-718 (1982)

Publisher's Note Springer Nature remains neutral with regard to jurisdictional claims in published maps and institutional affiliations. 